\title{
Mechanism Design with Information Acquisition
}

\author{
Sushil Bikhchandani • Ichiro Obara
}

Received: date / Accepted: date

\begin{abstract}
Consider a mechanism design setting in which agents acquire costly information about an unknown, payoff-relevant state of nature before participating in the mechanism. Information gathering is covert. We investigate conditions under which (i) efficient implementation and (ii) full surplus extraction are Bayesian incentive compatible and interim individually rational.
\end{abstract}

JEL Classification: D44, D82

Keywords Information acquisition · Full surplus extraction · Efficient implementation · Mechanism design · Bayesian implementation

\section{Introduction}

We consider a mechanism design setting in which multiple agents may acquire costly information about an unknown, payoff-relevant state of nature before participating in the mechanism. A mechanism proposed by the mechanism designer includes not only a social choice function (from agents' reported information to a social outcome) but also instructions from the mechanism designer to each agent as to how much information to acquire. Agents are endowed with private information and they may covertly acquire additional information at some cost before deciding whether to accept the mechanism.

We thank an anonymous referee for many useful suggestions. Any remaining errors are ours.

Ichiro Obara

405 Hilgard Ave., Los Angeles, CA 90095, USA

Tel.: +1-310-794-7098

Fax: +1-310-825-9528

E-mail: iobara@econ.ucla.edu

Sushil Bikhchandani

405 Hilgard Ave., Los Angeles, CA 90095, USA 
The mechanism designer implements an outcome based on agents' reported signals. An agent's utility is a function of the outcome, the state of nature, and the cost of acquired information. As the state of nature is not observed perfectly and every signal is informative about the state of nature, this leads to an interdependent values setting. We investigate conditions under which (i) efficient implementation and (ii) full surplus extraction are Bayesian incentive compatible and interim individually rational.

An example of our model is an auction where values have an unobservable common component. In most papers on auctions and on trading mechanisms, it is assumed that agents are endowed with some information and may not acquire any more. This assumption is implausible in many settings as agents can usually acquire information at a cost without being observed by other agents or the mechanism designer. ${ }^{1}$

A social choice rule recommends a profile of (simultaneous) information acquisitions to agents and maps agents' reported information to an outcome. A mechanism is a social choice rule together with a payment function that maps reported information to each agent's payment.

A social choice rule is ex post efficient if it selects an ex post efficient outcome after every realization of information at the recommended level of information acquisition. It is ex ante efficient if it is ex post efficient and recommends a level of information acquisition that maximizes the sum of (ex ante) expected utilities net of information acquisition costs. A mechanism fully extracts surplus if its social choice rule is efficient ${ }^{2}$ and each agent's interim expected surplus is zero. A social choice rule is implementable if it is a part of a mechanism that is Bayesian incentive compatible and interim individually rational.

Crémer and McLean [9] show that if agents are costlessly endowed with correlated information, then under some conditions on the information structure that are generically satisfied, full surplus extraction is Bayesian incentive compatible and interim individually rational. They provide two such conditions, the stronger one of which is the so called full-rank condition. In our setting with information acquisition, neither full surplus extraction nor efficiency is assured under their full-rank condition. We provide two examples to illustrate this: full-surplus extraction fails in one example and even efficient implementation fails in the other. In these examples, private signals are correlated and the full-rank condition of [9] is satisfied at all information acquisition levels.

We provide two sufficient conditions for efficient implementation. First, we show that efficient implementation is feasible for generic information structures when, for each agent, the set of other agents' endowed private signals is at least as large as either the set of states of nature that determine their utility or the

\footnotetext{
1 There are many papers on information acquisition in auction, but most of them focus on the private value case. Hausch and $\mathrm{Li}$ [18] studies information acquisition in a common-value auction.

2 From here on, efficiency means ex ante efficiency when describing a mechanism or a social choice rule. We will explicitly write ex post efficient when we mean efficiency in that sense.
} 
agent's signal space (Propositions 1 and 2). The efficient mechanism is a version of the expected externality mechanism (d'Aspremont and Gérard-Varet [10], Arrow [2]). ${ }^{3}$

Another sufficient condition for efficient implementation is the existence of semi-robust lotteries (Proposition 3). A set of semi-robust lotteries for an agent is a menu of payments such that acquiring more information than the recommended level would not help the agent make a better choice from the menu. We also provide sufficient conditions for the existence of semi-robust lotteries (Proposition 4).

Next, we show (in Proposition 5) that a sufficient condition for full surplus extraction is the existence of robust lotteries, which are semi-robust lotteries that are fair, i.e., give the agent an expected payoff of zero independent of his private information. A sufficient condition for the existence of robust lotteries for an agent is that the agent's signal space is no larger than either the set of states of nature or the set of other agents' endowed signals (Proposition 6).

With independent information, it is known that efficient implementation is Bayesian incentive compatible if and only if values are private (Bergemann and Välimäki [4], Stegeman [27]). With positively interdependent values and independent information, agents have an incentive to acquire more information than the socially optimal level (Bergemann, Shi and Välimäki [3], Bergemann and Välimäki [4]). The union of our sufficient conditions for efficient implementation covers the private value case (with independent or correlated information) as well as the case with interdependent values and correlated information. Our sufficient condition for full surplus extraction requires correlated information.

Although our primary focus is on the case with correlated information, our results are not a simple application of Crémer and McLean [9]. The fullrank condition of [9] is necessary for the existence of robust lotteries but not sufficient. Condition B of d'Aspremont and Gérard-Varet [11] is necessary and sufficient for balanced-budget implementation. Both [11] and [9] study environments without information acquisition. ${ }^{4}$ We discuss the relationship between semi-robust lotteries and Condition B of [11] in Section 4 and the connection between (semi-)robust lotteries and the full-rank condition of [9] in Sections 4 and 5.

Parreiras [25] shows that full surplus extraction may fail when each agent obtains two kinds of information exogenously: his payoff type and the informativeness of his type about the other agents' types. In our model, agents do not know their own type and acquire information about the types of all agents endogenously.

Obara [24] generalizes the model of Crémer and McLean [9] to a setting where agents take actions that change the distribution of their types that are payoff-relevant and finds a generic necessary and sufficient condition for full

\footnotetext{
3 We are not aware of any paper that uses an expected externality mechanism with correlated information and interdependent types.

4 See d'Aspremont, Crémer, and Gérard-Varet [12] for a survey of this literature.
} 
surplus extraction. Our paper generalizes [9] by allowing endogenous information acquisition, too. However, since we introduce a more specific structure to our model where payoffs and information about payoffs are distinguished, the necessary and sufficient condition in [24] on the joint distributions of private signals is never satisfied. Nonetheless, full surplus extraction is still possible in our setting because the set of possible utility functions is small as well.

Bikhchandani [6] shows that full surplus extraction fails if an agent can acquire costly information about other agents' types. ${ }^{5}$ Unlike in our paper, agents are fully and costlessly informed about their own type in [6]. Another difference is that in our paper the set of information signals available to each agent cannot be ranked by informativeness. Our sufficient conditions for efficient implementation are different from those in [6]. Robust lotteries are shown to be sufficient for full surplus extraction in [6]. Proposition 5 shows that robust lotteries are sufficient for full extraction in the more general model of this paper.

Neeman [23] notes that it is essential for the full-extraction result of Crémer and McLean that the belief of each agent pins down the payoff type of the agent, i.e., beliefs determine preferences (BDP property). This property is not necessarily satisfied in more general type spaces. ${ }^{6}$ Heifetz and Neeman [19] establish that the BDP property is non-generic in a geometric sense and a measure-theoretic sense in any convex family of (common) priors. On the other hand, Chen and Xiong [7] show that the set of priors on the universal type space where (almost) full surplus extraction is possible is topologically generic. Gizatulina and Hellwig [16] show that BDP property is topologically generic in finite dimensional type spaces.

The model is presented in Section 2. In Section 3 we provide examples showing the failure of full surplus extraction and of efficient implementation. Sufficient conditions for efficient implementation and for full surplus extraction are presented in Sections 4 and 5, respectively. We end with a discussion in Section 6 . Some proofs are in the appendix.

\section{The Model}

Consider a set of agents $N=\{1,2, \ldots, n\}, n \geq 2$. The state of nature $\omega \in \Omega$, where $\Omega$ is a finite set, determines agents' payoffs and is not observable, except perhaps after the end of the game. Importantly, the mechanism designer and the agents cannot contract on the state of nature. There is a common prior $q \in \Delta(\Omega)$, where $\Delta(\Omega)$ is the set of distributions on $\Omega$.

Agent $i$ can observe up to $K_{i}+1$ private signals $\tilde{s}_{i, k}, k \in Z_{i}=\left\{0,1, \ldots, K_{i}\right\}$. Each signal $\tilde{s}_{i, k}$ has support in a finite set $S_{i, k}$ for each $k$, where $\left|S_{i, k}\right| \geq 2$

\footnotetext{
5 Fang and Morris [13] show that the revenue equivalence theorem fails in an auction model in which each bidder observes a private signal about the other bidder's payoff type.

6 When BDP property is not satisfied, there may be two types whose belief types are the same, but payoff types are different. On the other hand, what causes a problem in [25] and [6] is the existence of two types with the same payoff and different beliefs. Thus, these papers provide somewhat complementary reasons for the failure of full surplus extraction.
} 
is assumed without loss of generality. These private signals are conditionally independent across players given state $\omega$. However, they may be correlated within each player. Formally, agent $i$ 's action set $A_{i}=2^{Z_{i}}$ is a collection of subsets of private signals. If agent $i$ chooses $a_{i} \in A_{i}$, then he observes a realization of $\tilde{s}_{i, k}$ if and only if $k \in a_{i}$ and he pays cost $c_{i}\left(a_{i}\right) \geq 0$. The cost $c_{i}$ is increasing in the sense that $c_{i}\left(a_{i}^{\prime}\right)>c_{i}\left(a_{i}\right)$ for any $a_{i}^{\prime}$ that is strictly larger than $a_{i}$ and $a_{i}^{\prime} \backslash a_{i}$ includes some $k \neq 0$. We assume that $c_{i}\left(a_{i} \bigcup\{0\}\right)=c_{i}\left(a_{i}\right)$ for any $a_{i}$. That is, agent $i$ always (costlessly) observes signal $\tilde{s}_{i, 0}$. Essentially, agent $i$ is endowed with signal $\tilde{s}_{i, 0}$. In the following, we assume without loss of generality that agent $i$ always observes $\tilde{s}_{i, 0}$ both in equilibrium and out of equilibrium.

The symbol $\tilde{s}^{a_{i}}$ denotes a vector of private signals $\left(\tilde{s}_{i, k}, k \in a_{i}\right)$. Also, $\tilde{s}_{-i}^{a_{-i}}=\left(\tilde{s}_{j}^{a_{j}}, j \neq i\right) .{ }^{7}$ We say that information is independent if and only if $\tilde{s}_{i}^{Z_{i}}, \tilde{s}_{j}^{Z_{j}}$, for all $i \neq j$, are independent random variables. Otherwise information is correlated. Let $p_{i}\left(s_{i}^{Z_{i}} \mid \omega\right)$ be the conditional probability distribution given $\tilde{\omega}=\omega$ of the signal $\tilde{s}_{i}^{Z_{i}}$, which takes values in the finite set $S_{i}^{Z_{i}}=S_{i, 0} \times \ldots \times S_{i, K_{i}}$. Note that $p_{i}$ is independent of agent $i$ 's action. Let $p$ be the collection of such conditional probability distributions $\left[p_{i}(\cdot \mid \omega), \forall \omega, \forall i\right]$. Let

$$
p_{i}^{a_{i}}\left(s_{i}^{a_{i}} \mid \omega\right):=\sum_{\substack{Z_{i} \backslash a_{i} \\ s_{i}}} p_{i}\left(s_{i}^{a_{i}}, s_{i}^{Z_{i} \backslash a_{i}} \mid \omega\right)
$$

be the probability distribution of $\tilde{s}_{i}^{a_{i}}$ given $\tilde{\omega}=\omega$ derived from $p$. Similarly, define

$$
p_{-i}^{a_{-i}}\left(s_{-i}^{a_{-i}} \mid \omega\right):=\Pi_{j \neq i} p_{j}^{a_{j}}\left(s_{j}^{a_{j}} \mid \omega\right)
$$

We make a full-support assumption: $p_{i}\left(s_{i}^{Z_{i}} \mid \omega\right)>0$ for every $s_{i}^{Z_{i}} \in S_{i}^{Z_{i}}, \omega \in \Omega$ and $i \in N$.

Given $q$ and $p_{i}$, agent $i$ 's posterior belief over $\tilde{\omega}=\omega$ conditional on observing $\tilde{s}_{i}^{a_{i}}=s_{i}^{a_{i}} \in S_{i}^{a_{i}}$ can be derived and is denoted by $d_{i}\left(\omega \mid s_{i}^{a_{i}}\right)$. The conditional probability distributions $d_{-i}\left(\omega \mid s_{-i}^{a_{-i}}\right)$ can be derived similarly from $q$ and $p_{-i}$. Each $d_{i}\left(\cdot \mid s_{i}^{a_{i}}\right)$ is a vector in $\mathbb{R}^{|\Omega|}$. Agent $i$ 's belief about the other agents' signals $\tilde{s}_{-i}^{a_{-i}}$ conditional on $\tilde{s}_{i}^{a_{i}}=s_{i}^{a_{i}}$ is

$$
h_{i}^{a_{-i}}\left(s_{-i}^{a_{-i}} \mid s_{i}^{a_{i}}\right):=\sum_{\omega \in \Omega} p_{-i}^{a_{-i}}\left(s_{-i}^{a_{-i}} \mid \omega\right) d_{i}\left(\omega \mid s_{i}^{a_{i}}\right)
$$

Let $X$ be a compact set of outcomes. The monetary transfer from agent $i$ to the mechanism designer is denoted $t_{i} \in \mathbb{R}$. Agent $i$ 's utility over outcome $x$, money transfer $t_{i}$, and information acquisition decision $a_{i}$ takes a quasi-linear form

$$
u_{i}(x, \omega)-t_{i}-c_{i}\left(a_{i}\right)
$$

7 For any vector $x=\left(x_{1}, \ldots, x_{n}\right), x_{-i}$ denotes $\left(x_{1}, \ldots, x_{i-1}, x_{i+1}, \ldots, x_{n}\right)$. The symbol $\tilde{x}$ denotes a random variable and $x$ is its realization. 
where $u_{i}(\cdot, \omega): X \rightarrow \mathbb{R}_{+}$is continuous for each $\omega \in \Omega$. Each agent has a large enough supply of the money commodity so that the budget constraint is not binding. is

Agent $i$ 's induced utility for outcome $x$ conditional on $s^{a}=\left(s_{1}^{a_{1}}, \ldots, s_{n}^{a_{n}}\right)$

$$
V_{i}\left(x, s^{a}\right):=E\left[u_{i}(x, \tilde{\omega}) \mid s^{a}\right]
$$

We say values are private if and only if $E\left[u_{i}(x, \tilde{\omega}) \mid s^{a}\right]$ does not depend on $s_{-i}^{a_{-i}}$ for any $i$ and any $a$. Otherwise values are interdependent. If $Z_{i}=\{0\}$ for each $i$, then agents cannot acquire any new information in addition to their endowed private information. Thus the model reduces to the model of Crémer and McLean [9] when the values are private and $Z_{i}=\{0\}$ for each $i$.

An information structure is a set of states, a set of signals, and a set of (conditional) distributions over states and signals: $\left(\Omega, S^{Z}, q, p\right)$, where $S^{Z}=$ $\left(S_{1}^{Z_{1}}, \ldots, S_{n}^{Z_{n}}\right)$. A mechanism design problem is an information structure together with an outcome set, utility functions, and cost functions: $\left(\Omega, S^{Z}, q, p, X, u, c\right)$ where $u=\left(u_{1}, \ldots, u_{n}\right)$ and $c=\left(c_{1}, \ldots, c_{n}\right)$.

Agent $i$ sends a message $m_{i} \in M_{i}$ upon choosing $a_{i}$ and observing $s_{i}^{a_{i}}$. Since we focus on pure strategy equilibrium, we can use $S_{i}^{a_{i}}$ as message space $M_{i}$ without loss of generality when $a_{i}$ is agent $i$ 's equilibrium action. ${ }^{8}$ A social choice function $f: S^{a} \rightarrow X$ maps agents' messages $s^{a}$ to outcome in $X$. A social choice rule $(a, f)$ is an information acquisition recommendation for each agent and a social choice function. A payment function $t: S^{a} \rightarrow \mathbb{R}^{n}$ maps agents' signals to transfers from the agents to the mechanism designer when $a$ is recommended. A mechanism $(a, f, t)$ is a social choice rule and a payment function $t=\left(t_{1}, \ldots, t_{n}\right)$.

The mechanism designer and agents play the following game. First, the mechanism designer proposes a mechanism $(a, f, t)$. As each agent $i$ is costlessly endowed with signal $\tilde{s}_{i, 0}$, in any mechanism we can assume $0 \in a_{i}$. Next, each agent $i$ covertly chooses an information acquisition level in $A_{i},{ }^{9}$ observes the corresponding private signal(s), and then decides whether to accept the mechanism. If he decides to accept, then agent $i$ reports a signal realization $s_{i}^{a_{i}} \in S_{i}^{a_{i}}$ to the mechanism designer (simultaneously with all the other agents). ${ }^{10}$ The mechanism designer implements the outcome $f\left(s^{a}\right)$ and collects transfers $t\left(s^{a}\right)$ based on the reported signal profile $s^{a} \in S^{a}$. We assume that the amount of information each agent acquires and whether or not each agent accepts the mechanism is not observable to the other agents. If an agent does not participate, his payoff, ignoring any information acquisition cost, is zero.

The assumption of conditional independence is not crucial for our results. In Proposition 3, 4, 5 and Corollary 1, the hypotheses are stated in terms of

\footnotetext{
8 See Proposition 1 in Obara [24].

9 Any information acquisition level selected by agent $i$ always includes his endowed signal 0 .

10 If agent $i$ reveals that he did not acquire the recommended information, either directly or indirectly by reporting $s_{i}^{\hat{a}_{i}} \notin S_{i}^{a_{i}}$, then the mechanism designer treats the agent as a non-participant.
} 
conditional distributions $h_{i}^{a_{-i}}\left(s_{-i}^{a_{-i}} \mid s_{i}^{a_{i}}\right)$ and $d_{i}\left(\omega \mid s_{i}^{a_{i}}\right), i \in N$. These results do not require independence of agents' private signals conditional on $\omega$, i.e., they hold for general joint distributions over $\Omega \times S^{Z}$. As Corollary 1 suggests, Proposition 1 and 2 can be modified to allow for private signals that are correlated conditional on $\omega$. The sufficient condition for Proposition 6 is even weaker when private signals are correlated.

We consider a pure-strategy perfect Bayesian equilibrium, where agents are sequentially rational given their subjective belief computed via Bayes' rule at all private histories. As we are interested in efficiency and full surplus extraction and $u_{i}$ is nonnegative for every agent, without loss of generality we restrict attention to mechanisms in which every agent always accepts the mechanism in equilibrium. ${ }^{11}$

\section{Bayesian Incentive Compatibility}

Bayesian incentive compatibility requires that each agent gathers exactly the amount of information specified by the mechanism designer and truthfully reports his signal. Suppose that the mechanism designer wants to implement a social choice rule $(a, f)$. The mechanism $(a, f, t)$ satisfies agent $i$ 's Bayesian incentive compatibility constraint if for all $\hat{a}_{i} \in A_{i}$

$$
\begin{aligned}
& E\left[V_{i}\left(f\left(\tilde{s}^{a}\right), \tilde{s}^{a}\right)-t_{i}\left(\tilde{s}^{a}\right)\right]-c_{i}\left(a_{i}\right) \\
\geq & \left.E\left[\max _{\max _{s_{i}} \in S_{i}^{a_{i}}} E\left[V_{i}\left(f\left(s_{i}^{a_{i}}, \tilde{s}_{-i}^{a_{-i}}\right),\left(s_{i}^{\hat{a}_{i}}, \tilde{s}_{-i}^{a_{-i}}\right)\right)-t_{i}\left(s_{i}^{a_{i}}, \tilde{s}_{-i}^{a_{-i}}\right) \mid \tilde{s}_{i}^{\hat{a}_{i}}=s^{\hat{a}_{i}}\right], 0\right\}\right]-c_{i}\left(\hat{a}_{i}\right)
\end{aligned}
$$

This constraint takes into account the possibility that an agent may take any information-gathering action and after observing his signal may not participate or may participate but lie about his signal. Note that even if agent $i$ takes an information-gathering action $\hat{a}_{i}$ different from $a_{i}$, the action specified by the mechanism designer, it is never in his interest to report a signal realization that is not in $S_{i}^{a_{i}}$, the support of $\tilde{s}_{i}^{a_{i}}$. If agent $i$ were to report a signal realization $\hat{s} \notin S_{i}^{a_{i}}$ then the mechanism designer concludes that agent $i$ did not follow his proposal to acquire $a_{i}$ and accordingly ignores agent $i$ 's signal and treats him as a non-participant (see footnote 10); the same outcome could be achieved by not participating, an option that agent $i$ has. Thus, without loss of generality, agent $i$ reports a signal $s_{i}^{a_{i}} \in S_{i}^{a_{i}}$ if he decides to participate.

The incentive compatibility constraint, (2), also implies interim individual rationality on the equilibrium path. To see this, consider a deviation where agent $i$ selects $a_{i}$ and opts out only when he observes a particular $s_{i}^{a_{i}}$ (otherwise agent $i$ participates and reports his signal truthfully), then (2) reduces to

$$
E\left[V_{i}\left(f\left(s_{i}^{a_{i}}, \tilde{s}_{-i}^{a_{-i}}\right),\left(s_{i}^{a_{i}}, \tilde{s}_{-i}^{a_{-i}}\right)\right)-t_{i}\left(s_{i}^{a_{i}}, \tilde{s}_{-i}^{a_{-i}}\right) \mid \tilde{s}_{i}^{a_{i}}=s_{i}^{a_{i}}\right] \geq 0, \quad \forall s_{i}^{a_{i}} \in S_{i}^{a_{i}}
$$

11 As agents' participation decisions are simultaneous and all agents participate in equilibrium, we do not need to explicitly consider the possibility that some agents do not participate. Thus we omit a detailed description of the mechanism after any non-participation. For completeness, one may assume that if one or more agents do not accept the mechanism, then an ex post efficient outcome for participating agents is implemented. 
Consider a mechanism design problem $\left(\Omega, S^{Z}, q, p, X, u, c\right)$ and a social choice rule $(a, f)$. If there exists $t$ such that $(2)$ is satisfied for each agent $i$ then $(a, f)$ can be implemented in this mechanism design problem. ${ }^{12}$

An ex post efficient social choice function given $a \in A$ is $f_{a}^{*}: S^{a} \rightarrow X$ that satisfies

$$
f_{a}^{*}\left(s^{a}\right) \in \arg \max _{x \in X} \sum_{i=1}^{n} V_{i}\left(x, s^{a}\right), \quad \forall s^{a} \in S^{a},
$$

which is well defined by the compactness of $X$ and continuity of $V_{i}$ with respect to $x$. Let

$$
V\left(a, s^{a}\right):=\sum_{i=1}^{n}\left[V_{i}\left(f_{a}^{*}\left(s^{a}\right), s^{a}\right)-c_{i}\left(a_{i}\right)\right],
$$

be the ex post maximized social surplus given $\left(a, s^{a}\right)$ and let $V(a):=E\left[V\left(a, \tilde{s}^{a}\right)\right]$ be the ex ante maximum social surplus given $a \in A$. Then, $a^{*}$ is an (ex ante) efficient information acquisition level if

$$
a^{*} \in \arg \max _{a \in A} V(a)
$$

Efficient implementation occurs when $\left(a^{*}, f_{a^{*}}^{*}\right)$ is implemented as an outcome of the Bayesian game induced by some mechanism $\left(a^{*}, f_{a^{*}}^{*}, t\right)$. Note that, if agent $i$ deviates to $a_{i} \neq a_{i}^{*}$, an ex post efficient outcome given $\left(a_{i}, a_{-i}^{*}\right)$ need not be implemented.

We consider two possible objectives for the mechanism designer: efficiency and profit maximization. The two objectives need not be in conflict and are simultaneously satisfied if the mechanism designer is able to implement an efficient social choice rule and extract the entire surplus.

\section{Two Examples}

If Crémer and McLean [9]'s full-rank condition or (weaker) cone condition is satisfied, then there exist lotteries (i.e., transfers) $t_{i}\left(s_{i}, s_{-i}\right)$ for each agent such that

$$
0=E\left[t_{i}\left(s_{i}, \tilde{s}_{-i}\right) \mid \tilde{s}_{i}=s_{i}\right]<E\left[t_{i}\left(\hat{s}_{i}, \tilde{s}_{-i}\right) \mid \tilde{s}_{i}=s_{i}\right], \quad \forall s_{i}, \forall \hat{s}_{i} \neq s_{i}
$$

The Crémer and McLean lotteries induce truth-telling in the absence of information acquisition.

In the first example below full surplus extraction is not possible, and in the second example efficient implementation (and therefore also full surplus extraction) is not possible. In these examples, Crémer and McLean [9] lotteries exist at every information acquisition level. Nonetheless, that does not prevent agents from acquiring more information than the efficient level.

\footnotetext{
12 We consider weak implementation, i.e., we only require that there exists an equilibrium that implements the desired social choice rule $(a, f)$.
} 
Example 1: FAILURE OF FULL SURPLUS EXTRACTION

There are two agents, $\alpha$ and $\beta$. A single indivisible object is allocated to either $\alpha$ or $\beta$. Agents have a common, unknown valuation $\tilde{\omega}$ for the object; $\tilde{\omega}$ takes values in the set $\Omega=\{0,2 \Delta, 3 \Delta\}$ where $\Delta>0$. Each of the three possible values of $\tilde{\omega}$ is equally likely.

Each agent $i$ costlessly observes signal $\tilde{s}_{i, 0} \in\{\ell, h\}$ about $\tilde{\omega}$. Agents' signals are independent conditional on $\tilde{\omega}$ and for $i=\alpha, \beta$,

$$
\begin{aligned}
& \operatorname{Pr}\left[\tilde{s}_{i, 0}=h \mid \tilde{\omega}=3 \Delta\right]=\operatorname{Pr}\left[\tilde{s}_{i, 0}=\ell \mid \tilde{\omega}=0\right]=q>0.5 \\
& \operatorname{Pr}\left[\tilde{s}_{i, 0}=h \mid \tilde{\omega}=2 \Delta\right]=0.5
\end{aligned}
$$

First, consider the scenario where agents do not have the option of acquiring additional information about $\tilde{\omega}$, other than the private signal that each costlessly observes. Because agents have common values, any rule that always allocates the object to, say, $\alpha$, is efficient. Then we can extract the full surplus by using Crémer and McLean lotteries.

Next, suppose that agent $i, i=\alpha, \beta$ has the option of acquiring, at cost $c>0$, a second signal $\tilde{s}_{i, 1}$ that reveals the true value of $\tilde{\omega}$. The common value assumption implies that it is not efficient for any agent to acquire this costly information. Therefore, in any full surplus extraction scheme, no agent must acquire the second signal.

Suppose that $(f, t)$ (with no acquisition of additional information) is a full surplus extraction mechanism. Since every allocation is efficient, we assume that the object is always allocated to agent $\alpha$ for simplicity. The same conclusion would be obtained based on similar arguments even if a more general social choice function is used.

Suppose that agent $\alpha$ deviates and covertly acquires the second signal at cost $c$. This second signal provides agent $\alpha$ with many options that he can exercise at the interim stage. For example, suppose that his expected payment conditional on $\tilde{\omega}=3 \Delta$ is more than $3 \Delta$. Then consider the following deviation: agent $\alpha$ acquires the second signal, does not participate in the mechanism if and only if $\tilde{\omega}=3 \Delta$, and follows the equilibrium strategy otherwise. The gain from this deviation is $q(3 \Delta)\left(E\left[t_{\alpha}(\tilde{s}) \mid \tilde{\omega}=3 \Delta\right]-3 \Delta\right)-c$, which must be nonpositive. Conversely, suppose that his expected payment conditional on $\tilde{\omega}=3 \Delta$ is less than $3 \Delta$. Then consider the following deviation: agent $\alpha$ acquires the second signal, participate in the mechanism (and follow the equilibrium) if and only if $\tilde{\omega}=3 \Delta$. The payoff from this deviation is $q(3 \Delta)\left(3 \Delta-E\left[t_{\alpha}(\tilde{s}) \mid \tilde{\omega}=3 \Delta\right]\right)-c$. This must be nonpositive as well because his equilibrium payoff is 0 by the assumption of full surplus extraction. Hence, noting that $q(3 \Delta)=\frac{1}{3}$, we obtain the following inequality:

$$
\left|E\left[t_{\alpha}(\tilde{s}) \mid \tilde{\omega}=3 \Delta\right]-3 \Delta\right| \leq 3 c
$$

This means that agent $\alpha$ 's expected payment must be almost equal to the object's value when he has the option of learning the true value and the cost 
of doing so is small. Similarly we can obtain the following inequality regarding $\tilde{\omega}=0$ :

$$
\left|E\left[t_{\alpha}(\tilde{s}) \mid \tilde{\omega}=0\right]\right| \leq 3 c
$$

Now consider the following deviation: agent $\alpha$ acquires the second signal, participate in the mechanism (and follow the equilibrium) if and only if $\tilde{\omega}=$ $2 \Delta$. Agent $\alpha$ 's payoff from this deviation is given by

$$
q(2 \Delta)\left(2 \Delta-E\left[t_{\alpha}(\tilde{s}) \mid \tilde{\omega}=2 \Delta\right]\right)-c
$$

Note that $E[\cdot \mid \tilde{\omega}=2 \Delta]=\frac{1}{2} E[\cdot \mid \tilde{\omega}=3 \Delta]+\frac{1}{2} E[\cdot \mid \tilde{\omega}=0]$ because of the structure of the signal distribution. Hence the above value is bounded below by

$$
\frac{1}{3}\left[2 \Delta-\left\{\frac{1}{2}(3 \Delta+3 c)+\frac{1}{2} 3 c\right\}\right]-c=\frac{0.5 \Delta-6 c}{3},
$$

which is strictly positive when $\Delta>12 c$. Hence there does not exist any full surplus extraction mechanism for this example when $\Delta$ is large relative to $c$.

It is clear that efficient implementation is possible in Example 1: randomly assign the object to either $\alpha$ or $\beta$ and do not collect any transfers. We turn to Example 2 for failure of efficient implementation.

\section{Example 2: FAILURE OF EFFICIENT IMPLEMENTATION}

There are two agents $\alpha$ and $\beta$. A single indivisible object is to be allocated to either $\alpha$ or $\beta$. Each agent's valuation is the sum of a private value and a common value:

$$
\tilde{V}_{i}=X_{i}(\tilde{\omega})+\tilde{V}, \quad i=\alpha, \beta
$$

The value of $X_{i}$ depends on whether the state $\tilde{\omega}$ is $\omega_{\alpha}$ or $\omega_{\beta}$. This dependence is shown in the table below.

\begin{tabular}{|c|c|c|}
\hline & $\omega_{\alpha}$ & $\omega_{\beta}$ \\
\hline$X_{\alpha}$ & 1 & 0 \\
\hline$X_{\beta}$ & 0 & $1+\epsilon$ \\
\hline
\end{tabular}

Each of the states $\omega_{\alpha}$ and $\omega_{\beta}$ is realized with probability 0.5 .

Each agent $i$ may observe two signals about $\tilde{\omega}: \tilde{s}_{i, 0} \in\left\{\omega_{\alpha}, \omega_{\beta}\right\}$ and $\tilde{s}_{i, 1} \in$ $\left\{\omega_{\alpha}, \omega_{\beta}\right\}$. The signal $\tilde{s}_{i, 0}$ is free and correct with probability $q>0.5$ (i.e., $\operatorname{Pr}\left(\tilde{s}_{i}=\omega_{y} \mid \tilde{\omega}=\omega_{y}\right)=q$, where $\left.y=\alpha, \beta\right)$. Moreover, $\tilde{s}_{\alpha, 0}$ and $\tilde{s}_{\beta, 0}$ are independent conditional on the true state $\omega$. The signal $\tilde{s}_{i, 1} \operatorname{costs} c_{\omega}>0$ and completely reveals the realization of the state $\tilde{\omega}$.

The common value $\tilde{V}$ is either 0 or $\Delta>0$, both equally likely. Moreover, the distribution of $\tilde{V}$ is independent of the state $\tilde{\omega}$ and $\tilde{s}_{0}:{ }^{13}$

$\operatorname{Pr}[\tilde{V}=\Delta]=\operatorname{Pr}\left[\tilde{V}=\Delta \mid \tilde{\omega}=\omega_{y}, \tilde{s}_{0}=\left(\omega_{y_{1}}, \omega_{y_{2}}\right)\right]=0.5, \quad \forall y, y_{1}, y_{2} \in\{\alpha, \beta\}$

13 This is not a critical assumption. We can use more general joint distributions at the cost of making the example more complex. 
Each agent can obtain perfect information about the realized value of $\tilde{V}$ at $\operatorname{cost} c_{v}>0$. However, acquisition of this costly information is inefficient as it does not increase allocative efficiency.

Assume that $c_{\omega}$ is large enough that it is not ex ante efficient for either agent to acquire $\tilde{s}_{i, 1}$. Further, assume that $\epsilon>0$ is small enough so that it is ex post efficient to allocate the object to agent $\alpha$ if and only if both agents' signals are $\omega_{\alpha}$. Finally, note that it is ex ante efficient that neither agent gathers costly information about $\tilde{V}$ as this is a common value. Thus none of the costly and perfectly informative signals $\left(\tilde{s}_{i, 1}\right.$ about $\tilde{\omega}$ and the signal about $\left.\tilde{V}\right)$ should be acquired by any agent for efficiency.

We show that this efficient rule is not Bayesian implementable.

Suppose, to the contrary, that there exists a Bayesian incentive-compatible efficient mechanism. By assumption, agent $\alpha$ truthfully reports $\tilde{s}_{\alpha, 0}$ without acquiring $\tilde{s}_{\alpha, 1}$ or information about $\tilde{V}$. That is, $\alpha$ follows the efficient rule.

Suppose that agent $\beta$ deviates and always announces $\omega_{\beta}$. Then he can win the object with probability 1 , while he would lose the object if $\tilde{s}_{0}=$ $\left(\omega_{\alpha}, \omega_{\alpha}\right)$ and he were to announce his signal truthfully. To deter this deviation, $E\left[t_{\beta}\left(\tilde{s}_{\alpha, 0}, \omega_{\beta}\right)-t_{\beta}\left(\tilde{s}_{\alpha, 0}, \omega_{\alpha}\right) \mid \tilde{s}_{\beta, 0}=\omega_{\alpha}\right]$ needs to be large enough. Thus, as $\Delta$ goes to infinity (recall that the size of $\Delta$ does not affect the efficient rule), $E\left[t_{\beta}\left(\tilde{s}_{\alpha, 0}, \omega_{\beta}\right)-t_{\beta}\left(\tilde{s}_{\alpha, 0}, \omega_{\alpha}\right) \mid \tilde{s}_{\beta, 0}=\omega_{\alpha}\right]$ must go to infinity as well for any mechanism that implements the efficient rule.

Now consider the following deviation by $\beta$. Agent $\beta$ acquires the signal about $V$, then lies by announcing $\omega_{\beta}$ only when $\tilde{s}_{\beta, 0}=\omega_{\alpha}$ and $V=\Delta$. Otherwise he always announces $\tilde{s}_{\beta, 0}$ truthfully. The incentive constraint regarding this deviation is as follows:

$$
\begin{aligned}
& \operatorname{Pr}\left(\tilde{s}_{\beta, 0}=\omega_{\alpha}\right)\left[q Q_{\alpha}\left(\omega_{\alpha}\right)+(1-q) Q_{\alpha}\left(\omega_{\beta}\right)\right] \\
\geq & \operatorname{Pr}\left(\tilde{s}_{\beta, 0}=\omega_{\alpha}\right)\left[q Q_{\beta}\left(\omega_{\alpha}\right)+(1-q) Q_{\beta}\left(\omega_{\beta}\right)\right] \\
& -\operatorname{Pr}\left(\tilde{s}_{\beta, 0}=\omega_{\alpha}\right) \operatorname{Pr}(\tilde{V}=0)\left[(1+\epsilon)(1-q)^{2}+E\left[t_{\beta}\left(\tilde{s}_{\alpha, 0}, \omega_{\alpha}\right)-t_{\beta}\left(\tilde{s}_{\alpha, 0}, \omega_{\beta}\right) \mid \tilde{s}_{\beta, 0}=\omega_{\alpha}\right]\right]-c_{v},
\end{aligned}
$$

where $Q_{y}\left(\omega_{z}\right)$ is $\beta$ 's expected payoff (including utility and payments) given that the true state is $\omega_{z}$ and $\beta$ announced $\omega_{y}$ for $y, z \in\{\alpha, \beta\}$.

Since $\operatorname{Pr}\left(\tilde{s}_{\beta, 0}=\omega_{\alpha}\right)=0.5$ and $\operatorname{Pr}(\tilde{V}=0)=0.5$, we have

$$
\begin{array}{r}
q\left[Q_{\alpha}\left(\omega_{\alpha}\right)-Q_{\beta}\left(\omega_{\alpha}\right)\right]+(1-q)\left[Q_{\alpha}\left(\omega_{\beta}\right)-Q_{\beta}\left(\omega_{\beta}\right)\right] \\
\geq 0.5 E\left[t_{\beta}\left(\tilde{s}_{\alpha, 0}, \omega_{\beta}\right)-t_{\beta}\left(\tilde{s}_{\alpha, 0}, \omega_{\alpha}\right) \mid \tilde{s}_{\beta, 0}=\omega_{\alpha}\right]-0.5(1+\epsilon)(1-q)^{2}-2 c_{v}
\end{array}
$$

We observe that the right-hand side of the inequality above increases without bound as $\Delta$ increases, thus the left-hand side increases without bound as $\Delta$ increases. Hence, either $Q_{\alpha}\left(\omega_{\alpha}\right)-Q_{\beta}\left(\omega_{\alpha}\right)$ or $Q_{\alpha}\left(\omega_{\beta}\right)-Q_{\beta}\left(\omega_{\beta}\right)$ goes to $\infty$ as $\Delta \rightarrow \infty$.

Therefore, it becomes profitable for agent $\beta$ to learn the true value of $\tilde{\omega}$ by acquiring the second signal $\tilde{s}_{\beta, 1}$. As this potential profit grows without bound as $\Delta$ increases and the cost of obtaining the second signal is constant, agent $\beta$ would prefer to obtain the second signal for a large enough $\Delta$. This proves that it is impossible to implement efficient social choice rule when $\Delta$ is large enough. 


\section{Efficient Bayesian Implementation}

In this section, we provide two complementary sufficient conditions for efficient implementation via pure-strategy perfect Bayesian equilibrium. The first one allows us to construct a version of the expected externality mechanism. The second sufficient condition is the existence of a semi-robust lottery. Because our setting allows information acquisition, this condition is not directly comparable to the existence of a full surplus extraction lottery in Crémer and McLean [9]. However, as discussed at the end of this section, in models without information acquisition, a sufficient condition for the existence of a semi-robust lottery is weaker than the necessary and sufficient condition (i.e. cone condition) for the existence of a full surplus extraction lottery.

Both sufficient conditions hold when, loosely speaking, each agent's signal space is not too large relative to the signal spaces of the other agents. ${ }^{14}$

\subsection{Expected Externality Mechanism}

Our first efficiency result provides a sufficient condition for the existence of a transfer for each agent that internalizes the expected externality of any action he takes on and off the equilibrium path given any signal he observes (when other agents take the equilibrium action).

We first define, for each agent $i$, a state space $\Omega_{-i}$ that is sufficient to describe the payoffs and information of the agents other than $i$. To be precise, we define $\Omega_{-i}$ as the coarsest partition of $\Omega$, the state space of the model, such that $\sum_{j \neq i} u_{j}(\cdot, \omega)$ and $p_{-i}(\cdot \mid \omega)$ are invariant on each element of the partition. For example, consider a private value model where $\Omega=\Omega_{1} \times \cdots \times \Omega_{n}$ and $\omega_{j} \in \Omega_{j}$ only affects $u_{j}$ and the distribution of $\tilde{s}_{j}^{Z_{j}}$. Then $\Omega_{-i}$ is simply $\Pi_{j \neq i} \Omega_{j}$.

Let $d_{i}^{\prime}\left(\cdot \mid s_{i}^{a_{i}}\right)$ and $d_{-i}^{\prime}\left(\cdot \mid s_{-i}^{a_{-i}}\right)$ be the conditional distributions on $\Omega_{-i}$ that are derived from $d_{i}\left(\cdot \mid s_{i}^{a_{i}}\right)$ and $d_{-i}\left(\cdot \mid s_{-i}^{a_{-i}}\right)$ respectively. Let $M_{i}^{Z_{i}}$ be a basis of the subspace in $\mathbb{R}^{\left|\Omega_{-i}\right|}$ that is orthogonal to the subspace spanned by $\left\{d_{i}^{\prime}\left(\cdot \mid s_{i}^{Z_{i}}\right) \mid s_{i}^{Z_{i}} \in S_{i}^{Z_{i}}\right\}$, i.e., $d_{i}^{\prime}\left(\cdot \mid s_{i}^{Z_{i}}\right) \cdot \gamma=0$ for every $s_{i}^{Z_{i}} \in S_{i}^{Z_{i}}$ and every $\gamma \in M_{i}^{Z_{i}}$. Observe that $\left|M_{i}^{Z_{i}}\right| \geq \max \left\{\left|\Omega_{-i}\right|-\left|S_{i}^{Z_{i}}\right|, 0\right\}$, with equality for generic $(q, p)$.

Proposition 1 Let $a^{*} \in A$ be an efficient information acquisition profile for a mechanism design problem $\left(\Omega, S^{Z}, q, p, X, u, c\right)$. Suppose that for every $i \in N$, a subset of vectors in $\left\{d_{-i}^{\prime}\left(\cdot \mid s_{-i}^{a_{-i}^{*}}\right), s_{-i}^{a_{-i}^{*}} \in S_{-i}^{a_{-i}^{*}}\right\}$ and $M_{i}^{Z_{i}}$ form a basis for $\mathbb{R}^{\left|\Omega_{-i}\right|}$. Then $a^{*}$ and the ex post efficient social choice function $f_{a^{*}}^{*}$ can be implemented.

14 McLean and Postlewaite [22] discusses a notion of information size. However, their notion of information size is not directly relevant here. 
Proof: In the following, it is assumed that no agent opts out. This is without loss of generality because one can decrease transfers by a constant so that all the participation constraints are satisfied.

First, it is shown that there exists $t_{i}: S^{a^{*}} \rightarrow \mathbb{R}$, a transfer function for agent $i$, that satisfies (5) below, for every $\widehat{s}_{i}^{a_{i}^{*}} \in S_{i}^{a_{i}^{*}}, s_{i}^{Z_{i}}=\left(s_{i}^{a_{i}^{*}}, s_{i}^{Z_{i} \backslash a_{i}^{*}}\right) \in S_{i}^{Z_{i}}$, where it is possible that $\widehat{s}_{i}^{a_{i}^{*}} \neq s_{i}^{a_{i}^{*}}$ :

$$
\begin{aligned}
& \sum_{\omega_{-i} \in \Omega_{-i}} E\left[t_{i}\left(\widehat{s}_{i}^{a_{i}^{*}}, \tilde{s}_{-i}^{a_{-i}}\right) \mid \omega_{-i}\right] d_{i}^{\prime}\left(\omega_{-i} \mid s_{i}^{Z_{i}}\right) \\
= & \sum_{\omega_{-i} \in \Omega_{-i}} E\left[\sum_{j \neq i}-u_{j}\left(f_{a^{*}}^{*}\left(\widehat{s}_{i}^{a_{i}^{*}}, \tilde{s}_{-i}^{a_{-i}^{*}}\right), \omega_{-i}\right) \mid \omega_{-i}\right] d_{i}^{\prime}\left(\omega_{-i} \mid s_{i}^{Z_{i}}\right)
\end{aligned}
$$

Let $D_{i}$ be a $\left|S_{i}^{Z_{i}}\right| \times\left|\Omega_{-i}\right|$ matrix, each row of which is the vector $d_{i}^{\prime}\left(\cdot \mid s_{i}^{Z_{i}}\right)$ for some $s_{i}^{Z_{i}} \in S_{i}^{Z_{i}}$. Let $P_{-i}$ be a $\left|\Omega_{-i}\right| \times\left|S_{-i}^{a_{-i}^{*}}\right|$ matrix, each row of which is

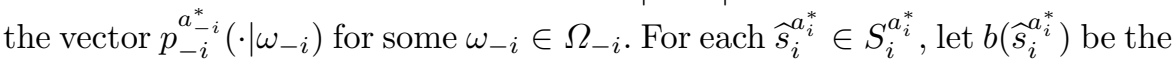
$\left|\Omega_{-i}\right|$-dimensional column vector that has $E\left[-\sum_{j \neq i} u_{j}\left(f_{a^{*}}^{*}\left(\widehat{s}_{i}^{a_{i}^{*}}, \tilde{s}_{-i}^{a_{-i}^{*}}\right), \omega_{-i}\right) \mid \omega_{-i}\right]$ as its $\omega^{\text {th }}$ element. Then, a function $t_{i}$ satisfying (5) is a solution to the following system of equations for each $\widehat{s}_{i}^{a_{i}^{*}}$ :

$$
D_{i} P_{-i} x=D_{i} b\left(\widehat{s}_{i}^{*}\right)
$$

Let $\Gamma_{i}$ be a $\left|\Omega_{-i}\right| \times\left|M_{i}^{Z_{i}}\right|$ matrix with the vectors in $M_{i}^{Z_{i}}$ as its column vectors. By assumption, the number of independent column vectors of $P_{-i}$ and $\Gamma_{i}$ is $\left|\Omega_{-i}\right|{ }^{15}$ Hence there exists $x^{\prime} \in \mathbb{R}^{\left|S_{-i}^{a_{-i}^{*}}\right|}, z \in \mathbb{R}^{\left|M_{i}^{Z_{i}}\right|}$ such that $b\left(\widehat{s}_{i}^{a_{i}^{*}}\right)=$ $P_{-i} x^{\prime}+\Gamma_{i} z$. This $x^{\prime}$ is a solution for $(6)$ because $D_{i} \Gamma_{i}=0$ by definition.

Next, it is verified that $a_{i}^{*}$ and $f_{a^{*}}^{*}$ can be implemented with the transfer $t_{i}$ just defined. The optimality of $a_{i}^{*}$ and truthful reporting follow from the fact that, as can be seen in $(5), \sum_{\omega_{-i} \in \Omega_{-i}} E\left[-t_{i}\left(\widehat{s}_{i}^{a_{i}^{*}}, \tilde{s}_{-i}^{a_{-i}^{*}}\right) \mid \omega_{-i}\right] d_{i}^{\prime}\left(\omega_{-i} \mid s_{i}^{Z_{i}}\right)$ is exactly the total expected utility of the agents other than agent $i$ given $i$ 's report $\widehat{s}_{i}^{a_{i}^{*}} \in S_{i}^{a_{i}^{*}}$ conditional on any $s_{i}^{Z_{i}} \in S_{i}^{Z_{i}}$. This means that the expected externality of agent $i$ 's report on other agents given any $s_{i}^{Z_{i}} \in S_{i}^{Z_{i}}$ can be internalized through transfer $t_{i}$ on and off the equilibrium path without eliciting agent $i$ 's true signals.

For example, suppose that agent $i$ acquires all $K_{i}$ signals. Then agent $i$ 's expected payoff conditional on observing $s_{i}^{Z_{i}} \in S_{i}^{Z_{i}}$ and reporting $\widehat{s}_{i}^{a_{i}^{*}} \in S_{i}^{a_{i}^{*}}$ is

$$
\sum_{\omega} E\left[u_{i}\left(f_{a^{*}}^{*}\left(\widehat{s}_{i}^{a_{i}^{*}}, \tilde{s}_{-i}^{a_{-i}^{*}}\right), \omega\right)-t_{i}\left(\widehat{s}_{i}^{*}, \tilde{s}_{-i}^{a_{-i}^{*}}\right) \mid \omega\right] d_{i}\left(\omega \mid s_{i}^{Z_{i}}\right)
$$

15 The rank of $P_{-i}$ is the same as the rank of $\left\{d_{-i}^{\prime}\left(\cdot \mid s_{-i}^{a_{-i}^{*}}\right), s_{-i}^{a_{-i}^{*}} \in S_{-i}^{a_{-i}^{*}}\right\}$. To see this, note that $q\left(\omega_{-i}\right) p_{-i}^{a_{-i}^{*}}\left(s_{-i}^{a_{-i}^{*}} \mid \omega_{-i}\right) \propto d_{-i}^{\prime}\left(\omega_{-i} \mid s_{-i}^{a_{-i}^{*}}\right)$ by Bayes' rule. 


$$
\begin{aligned}
& =\sum_{\omega} E\left[u_{i}\left(f_{a^{*}}^{*}\left(\widehat{s}_{i}^{a_{i}^{*}}, \tilde{s}_{-i}^{a_{-i}^{*}}\right), \omega\right) \mid \omega\right] d_{i}\left(\omega \mid s_{i}^{Z_{i}}\right)-\sum_{\omega_{-i}} E\left[t_{i}\left(\widehat{s}_{i}^{a_{i}^{*}}, \tilde{s}_{-i}^{a_{-i}^{*}}\right) \mid \omega_{-i}\right] d_{i}^{\prime}\left(\omega_{-i} \mid s_{i}^{Z_{i}}\right) \\
& =\sum_{\omega} E\left[u_{i}\left(f_{a^{*}}^{*}\left(\widehat{s}_{i}^{a_{i}^{*}}, \tilde{s}_{-i}^{a_{-i}^{*}}\right), \omega\right) \mid \omega\right] d_{i}\left(\omega \mid s_{i}^{Z_{i}}\right)-\sum_{\omega_{-i}} E\left[\sum_{j \neq i}-u_{j}\left(f_{a^{*}}^{*}\left(\widehat{s}_{i}^{a_{i}^{*}}, \tilde{s}_{-i}^{a_{-i}^{*}}\right), \omega_{-i}\right) \mid \omega_{-i}\right] d_{i}^{\prime}\left(\omega_{-i} \mid s_{i}^{Z_{i}}\right) \\
& =\sum_{\omega} E\left[\sum_{j=1}^{n} u_{j}\left(f_{a^{*}}^{*}\left(\widehat{s}_{i}^{*}, \tilde{s}_{-i}^{a_{i}^{*}}, \omega\right) \mid \omega\right] d_{i}\left(\omega \mid s_{i}^{Z_{i}}\right),\right.
\end{aligned}
$$

by the definition of $t_{i}$. Note that this is the expected social welfare (without the information acquisition cost) of allocation $f_{a^{*}}^{*}\left(\widehat{s}_{i}^{a_{i}^{*}}, \cdot\right)$ given $s_{i}^{Z_{i}} \in S_{i}^{Z_{i}}$. Since $f_{a^{*}}^{*}$ does not take into account agent $i$ 's true action $\left(a_{i}=Z_{i}\right)$, it does not maximize the social welfare given $\left(Z_{i}, a_{-i}^{*}\right)$. Hence agent $i$ 's ex ante expected payoff when choosing $\left(Z_{i}, a_{-i}^{*}\right)$ is bounded above by $V\left(Z_{i}, a_{-i}^{*}\right)+\sum_{j \neq i} c_{j}\left(a_{j}^{*}\right)$.

Similarly it can be shown that (i) agent $i$ 's ex ante expected payoff conditional on $\left(a_{i}, a_{-i}^{*}\right)$ for any $a_{i} \neq a_{i}^{*}$ is at most $V\left(a_{i}, a_{-i}^{*}\right)+\sum_{j \neq i} c_{j}\left(a_{j}^{*}\right)$ and (ii) agent $i$ can achieve $V\left(a^{*}\right)+\sum_{j \neq i} c_{j}\left(a_{j}^{*}\right)$ exactly by choosing $a_{i}^{*}$ and always announcing his signal truthfully. Since $a^{*}$ is the action profile that maximizes the social welfare, it is optimal for agent $i$ to play $a_{i}^{*}$ and report his signal truthfully under transfer function $t_{i}$. Hence social choice rule $\left(a^{*}, f_{a^{*}}^{*}\right)$ can be implemented by mechanism $\left(a^{*}, f_{a^{*}}^{*}, t\right)$.

If $\omega$ is not observable ex post, then we may like to find a sufficient condition on the distribution of signals only. A condition on $h_{i}^{a_{-i}^{*}}$ that is stronger than the sufficient condition for Proposition 1, hence guarantees efficient implementation, is provided in Corollary 1. The proof is in the appendix.

Corollary 1 Let $a^{*} \in A$ be an efficient information acquisition profile for a mechanism design problem $\left(\Omega, S^{Z}, q, p, X, u, c\right)$. Suppose that for every $i \in N$, the set of vectors $\left\{h_{i}^{a_{-i}^{*}}\left(\cdot \mid s_{i}^{Z_{i}}\right), s_{i}^{Z_{i}} \in S_{i}^{Z_{i}}\right\}$ has rank $\left|S_{i}^{Z_{i}}\right|$. Then $a^{*}$ and the ex post efficient allocation $f_{a^{*}}^{*}$ can be implemented.

\section{Remark 1}

(i) This mechanism builds on the idea of internalizing externality through transfers, like many well-known mechanisms such as the VCG mechanism (Vickrey [28], Clarke [8], Gloves [17] and the expected externality mechanism (d'Aspremont and Gérard-Varet [10], Arrow [2]).

(ii) Proposition 1 holds as stated even if agent $i$ 's utility is directly affected by his action $\left(u_{i}\left(x, \omega, a_{i}\right)\right)$.

(iii) Corollary 1 holds even if private signals are not conditionally independent.

(iv) The model assumes that each agent chooses a set of signals once and for all simultaneously. We can also think of situations where each agent chooses signals sequentially. Then an agent may decide which costly signal he wishes to acquire next based on the realization of signals he has observed previously without knowing the information acquired by the other agents. Call this sequential information acquisition within agents. We can define 
efficient information acquisition rule within this class of sequential information acquisitions. Such sequential information acquisition improves efficiency, but Proposition 1 still extends to this case almost immediately. For within-agents sequential information acquisition, an efficient information acquisition rule is defined as a profile of contingent strategies $\left(\sigma_{1}^{*}, \ldots, \sigma_{n}^{*}\right)$. Let $S_{j}^{\sigma_{j}^{*}}$ be the set of all possible signal realizations for agent $j$ given $\sigma_{j}^{*}$. Proposition 1 holds in this case if we replace $s_{-i}^{a_{-i}^{*}}$ with $s_{-i}^{\sigma_{-i}^{*}}$.

To understand the condition for Proposition 1, consider the standard model with no information acquisition (i.e. $Z_{i}=\{0\}$ ). Let $\Omega=\Omega_{1} \times \ldots \times \Omega_{n}$, where $\omega_{i}$ completely determines agent $i$ 's utility function and the distribution of $s_{i, 0}$. Then the following two familiar conditions imply the condition for Proposition 1. First, the condition is satisfied with independent private values (i.e. agent $i$ 's signal $s_{i, 0}$ does not depend on $\omega_{-i}$ and $\left(\omega_{1}, \ldots, \omega_{n}\right)$ are independent). ${ }^{16}$ In this case, $M_{i}^{Z_{i}}$ is a set of $\left|\Omega_{-i}-1\right|$ independent vectors that are orthogonal to agent $i$ 's prior about $\omega_{-i}$, which is a convex combination of the posteriors about $\omega_{-i}$ given the private signals of all the agents other than agent $i$. Hence those posteriors and $M_{i}^{Z_{i}}$ span $R^{\left|\Omega_{-i}\right|}$. Second, the condition is satisfied when the information structure satisfies the full-rank condition in Crémer and McLean [9], which requires $\left\{h_{i}^{a^{*}}\left(\cdot \mid s_{i}^{Z_{i}}\right), s_{i}^{Z_{i}} \in S_{i}^{Z_{i}}\right\}$ to be independent vectors. As already mentioned, this condition is stronger than the condition for Proposition 1 as shown in the proof of Corollary $1 .^{17}$

Efficient implementation result for the independent private value case remains valid even with information acquisition. As long as agent $i$ 's signal is not informative about $\omega_{-i}$ given any information acquisition level, the dimension of $M_{i}^{Z_{i}}$ is $\left|\Omega_{-i}-1\right|$. Thus efficient implementation is always possible in private values models with information acquisition. This replicates the result of Bergemann and Välimäki [4] in a more general setting.

It is known that, with information acquisition, efficient implementation is impossible for the case with interdependent value and independent information. ${ }^{18}$ Proposition 1 shows that efficient implementation is possible when values are interdependent and information is correlated.

Neither the VCG mechanism nor the expected externality mechanism guarantees efficiency in the standard interdependent value model. This is because agent $i$ 's true signal needs to be elicited to evaluate the externality of agent $i$ 's report on the other agents. In our setting, this corresponds to the fact that $\sum_{j \neq i} V_{j}\left(x, s^{a}\right)$ depends on $s_{i}^{a_{i}}$. So we cannot internalize such externality for each realization of $s^{a}$. However, a crucial observation for our result is that the

\footnotetext{
16 The signal $s_{i, 0}$ is often $\omega_{i}$ itself in the standard model, including the model of Crémer and McLean [9].

17 The weaker condition (cone condition) in Crémer and McLean [9] is not directly comparable to this condition.

18 This follows from the revenue equivalence theorem and the negative result of [4], as noted in Bergemann, Shi, and Välimäki [3]. So we need correlation of private signals for our efficient implementation result when values are interdependent
} 
expected externality conditional on $\omega_{-i}$, which is $E\left[\sum_{j \neq i} u_{j}\left(x\left(\widehat{s}_{i}^{a_{i}}, \tilde{s}_{-i}^{a_{-i}}\right), \omega_{-i}\right) \mid \omega_{-i}\right]$, can be evaluated without eliciting agent $i$ 's signal. Although $\omega_{-i}$ is not available, we can use the signals of the agents other than agent $i$ to replicate this value in expectation. If $\left\{d_{-i}^{\prime}\left(\cdot \mid s_{-i}^{a_{-i}^{*}}\right), s_{-i}^{a_{-i}^{*}} \in S_{-i}^{a_{-i}^{*}}\right\}$ form a basis for $\mathbb{R}^{\left|\Omega_{-i}\right|}$ by themselves, we can in fact replicate these expected values exactly state by state, i.e., there exists $t_{i}$ that satisfies, for any $\omega_{-i}$ and any $\widehat{s}_{i}^{a_{i}^{*}}$,

$$
E\left[t_{i}\left(\widehat{s}_{i}^{a_{i}^{*}}, \tilde{s}_{-i}^{a_{-i}^{*}}\right) \mid \omega_{-i}\right]=E\left[-\sum_{j \neq i} u_{j}\left(f_{a^{*}}^{*}\left(\widehat{s}_{i}^{*}, \tilde{s}_{-i}^{a_{-i}^{*}}\right), \omega_{-i}\right) \mid \omega_{-i}\right]
$$

This is why our mechanism relies on expected externality rather than exact externality. ${ }^{19}$

The condition for Proposition 1 is weaker than this. More generally, $\left\{d_{-i}^{\prime}\left(\cdot \mid s_{-i}^{a_{-i}^{*}}\right), s_{-i}^{a_{-i}^{*}} \in S_{-i}^{a_{-i}^{*}}\right\}$ does not have to span $\mathbb{R}^{\left|\Omega_{-i}\right|}$. Notice that agent $i$ 's transfer does not need to replicate the expected externality state by state exactly as long as it replicates the expected externality at the interim stage conditional on $i$ 's signal. This can be done more easily when the size of $\left|S_{i}^{Z_{i}}\right|$ is relatively small and/or some of agent $i$ 's signals are redundant (i.e. not independent), in which case $M_{i}^{Z_{i}}$ is large. Thus, the rank of $M_{i}^{Z_{i}}$ also matters for efficient implementation.

The next proposition makes this observation formal by showing that efficient implementation is possible for a generic choice of $(q, p)$ under the corresponding conditions on the size of signal spaces. Note that the parameter space of $(q, p)$ is finite dimensional because the number of states and signals is finite. Let $S_{-i}^{0}=\prod_{j \neq i} S_{j}^{0}$ be the space of free signals $s_{j}^{0}, j \neq i$.

Proposition 2 Fix $\left(\Omega, S^{Z}, X, u, c\right)$. If $\left|S_{-i}^{\mathbf{0}}\right| \geq \min \left\{|\Omega|,\left|S_{i}^{Z_{i}}\right|\right\}$ for each $i$, then efficient implementation is possible for mechanism design problem $\left(\Omega, S^{Z}, q, p, X, u, c\right)$ for an open and dense set of $(q, p)$.

Proof: We can restrict our attention to the case where $q$ has full support for this genericity result.

First, consider the case where $\left|S_{-i}^{\mathbf{0}}\right|$ is larger than or equal to $|\Omega|$. Consider a set of $(q, p)$ where $\left\{p_{-i}^{\mathbf{0}}(\cdot \mid \omega), \omega \in \Omega\right\}$ is linearly independent. Note that $\Omega_{-i}$ is $\Omega$ itself in this set by the definition of $\Omega_{-i}$. Since $\left\{p_{-i}^{a_{-i}^{*}}(\cdot \mid \omega), \omega \in \Omega\right\}$ is linearly independent in this set whatever the efficient action profile $a_{-i}^{*}$ is, Proposition 1 applies at least for player $i$. We show that this set is open and dense.

\footnotetext{
19 In d'Aspremont and Gérard-Varet [10], an externality mechanism provides incentives for efficient implementation by internalizing the externality; an expected externality mechanism delivers budget balance in addition. On the other hand, we need to use an expected externality mechanism even to internalize the externality.
} 
Openness is trivial. To show that this set is dense, take any $(q, p)$. Take $\widehat{p}$ such that $\left\{\widehat{p}_{-i}^{\mathbf{0}}(\cdot \mid \omega), \omega \in \Omega\right\}$ is linearly independent. Define $p(t), t \in[0,1]$ by $p(t)(\cdot \mid \omega):=t \widehat{p}(\cdot \mid \omega)+(1-t) p(\cdot \mid \omega)$ for each $\omega \in \Omega$. We have a one-parameter family of conditionally independent distributions that connect $\widehat{p}$ and $p$. Then it can be shown that $\left\{p(t)_{-i}^{\mathbf{0}}(\cdot \mid \omega), \omega \in \Omega\right\}$ is linearly independent for almost all $t$ (Fudenberg, Levine and Maskin [14]). Hence we can find $\widetilde{p}$ arbitrary close to $p$ such that $\left\{\widetilde{p}_{-i}^{\mathbf{0}}(\cdot \mid \omega), \omega \in \Omega\right\}$ is linearly independent. This proves denseness.

The case where $\left|S_{-i}^{\mathbf{0}}\right|<|\Omega|$ but $\left|S_{-i}^{\mathbf{0}}\right| \geq\left|S_{i}^{Z_{i}}\right|$ is similar. Under this assumption, we can show in a similar way that the rank of $\left\{d_{-i}\left(\cdot \mid s_{-i}^{\mathbf{0}}\right), s_{-i}^{\mathbf{0}} \in S_{-i}^{\mathbf{0}}\right\}$ is $\left|S_{-i}^{\mathbf{0}}\right|$ and the rank of $M_{i}^{Z_{i}}$ is at least $\max \left\{|\Omega|-\left|S_{i}^{Z_{i}}\right|, 0\right\}$ for an open dense set of $(q, p)$. Since $\left|S_{i}^{Z_{i}}\right| \leq\left|S_{-i}^{\mathbf{0}}\right|<|\Omega|$ by assumption, we have $\left|S_{-i}^{\mathbf{0}}\right|+$ $|\Omega|-\left|S_{i}^{Z_{i}}\right| \geq|\Omega|$. Then generically there are $|\Omega|$ independent vectors among $\left\{d_{-i}\left(\cdot \mid s_{-i}^{\mathbf{0}}\right), s_{-i}^{\mathbf{0}} \in S_{-i}^{\mathbf{0}}\right\}$ and $M_{i}^{Z_{i}}$. Hence generically there are $|\Omega|$ independent vectors among $\left\{d_{-i}\left(\cdot \mid s_{-i}^{a_{-i}^{*}}\right), s_{-i}^{a_{-i}^{*}} \in S_{-i}^{a_{-i}^{*}}\right\}$ and $M_{i}^{Z_{i}}$ whatever the efficient action profile $a^{*}$ is, thus Proposition 1 applies for player $i$.

\subsection{Semi-Robust Lotteries}

Next we turn to a complementary sufficient condition under which efficient implementation is obtained. Our second sufficient condition allows us to enforce any action and any allocation independent of the shape of utility functions. In that sense the analysis is very different from that of Section 4.1.

Following Crémer and McLean [9], it is useful to view transfers as a lottery by which an agent, based on his information, bets on the announcements of other agents. A set of lotteries is semi-robust for agent $i$ given $a$ if agent $i$ cannot make a better choice by acquiring more information than $a_{i}$. More precisely, $t_{i}: S^{a} \rightarrow \mathbb{R}$ is semi-robust given a for agent $i$ if

$$
\sum_{\substack{a_{-i} \\ s_{-i}}} t_{i}\left(s_{i}^{a_{i}}, s_{-i}^{a_{-i}}\right) h_{i}^{a_{-i}}\left(s_{-i}^{a_{-i}} \mid s_{i}^{a_{i}}, s_{i}^{Z_{i} \backslash a_{i}}\right)<\sum_{\substack{a_{-i} \\ s_{-i}}} t_{i}\left(\widehat{s}_{i}^{a_{i}}, s_{-i}^{a_{-i}}\right) h_{i}^{a_{-i}}\left(s_{-i}^{a_{-i}} \mid s_{i}^{a_{i}}, s_{i}^{Z_{i} \backslash a_{i}}\right),
$$

for any $s_{i}^{a_{i}}, \widehat{s}_{i}^{a_{i}} \neq s_{i}^{a_{i}} \in S_{i}^{a_{i}}$ and any $s_{i}^{Z_{i} \backslash a_{i}} \in S_{i}^{Z_{i} \backslash a_{i}}$. Thus, if agent $i$ takes action $a_{i}$ and observes $s_{i}^{a_{i}} \in S_{i}^{a_{i}}$, then it is optimal for him to choose $t_{i}\left(s_{i}^{a_{i}}, \cdot\right)$ from the set of lotteries $\left\{t_{i}\left(\widehat{s}_{i}^{a_{i}}, \cdot\right): \widehat{s}_{i}^{a_{i}} \in S_{i}^{a_{i}}\right\}$. Furthermore, this optimal choice of lottery would not change even if agent $i$ acquires more information.

By using semi-robust lotteries for Bayesian implementation, we can discourage agents from acquiring more information or from acquiring less information than the target level. It does not pay for an agent to acquire more information because it provides no gain in the mechanism and the cost of additional information is not reimbursed. It does not pay to acquire less in- 
formation because then the agent would be likely to make a wrong choice of semi-robust lottery.

Proposition 3 Consider an information structure $\left(\Omega, S^{Z}, q, p\right)$ and an informationacquisition decision $a=\left(a_{1}, a_{2}, \ldots, a_{n}\right)$. Suppose that for each agent $i$ there exist semi-robust lotteries given a. Then for any mechanism design problem on this information structure and any social choice function $f$, the social choice rule $(a, f)$ can be implemented.

Proof: Let $t_{i}^{\prime}$ be semi-robust lotteries for agent $i$ given $a$. We show that if, for some large $\lambda>0$, monetary transfers $t_{i}=\lambda t_{i}^{\prime}$ are used then it is Bayesian incentive compatible for agent $i$ to acquire information $a_{i}$ and truthfully report his signal.

Participation Constraints: We can assume without loss of generality that $t_{i}$ is non-positive and that agent $i$ 's expected surplus under truthful participation is non-negative. Hence agent $i$ would accept the mechanism $(a, f, t)$.

Deviation to ACQUiRe mORE INFORMATION: Suppose that $a_{i}$ is a strict subset of $Z_{i}$ and agent $i$ chooses information acquisition $Z_{i}$ and observes $s_{i}^{Z_{i}}=$ $\left(s_{i}^{a_{i}}, s_{i}^{Z_{i} \backslash a_{i}}\right)$. As $t_{i}$ is semi-robust given $a$, the expected amount of transfer from agent $i$ increases strictly by announcing any $\widehat{s}_{i}^{a_{i}} \neq s_{i}^{a_{i}}$, whatever agent $i$ 's additional information $s_{i}^{Z_{i} \backslash a_{i}}$ may be. Agent $i$ may gain some payoff by announcing $\widehat{s}_{i}^{a_{i}}$ instead of $s_{i}^{a_{i}}$ through changing the final allocation $f\left(s^{a}\right)$. But because the number of signals is finite we can choose $\lambda$ large enough so that this effect is outweighed by the expected loss in transfers. The same reasoning applies to any deviation to a superset of $a^{i}$. Hence, agent $i$ does not gain anything by acquiring more information. In fact agent $i$ is worse off as he is not reimbursed for the cost of acquiring additional information.

DEVIATION TO ACQUIRE LESS OR DIFFERENT INFORMATION Suppose that agent $i$ chooses action $\hat{a}_{i} \in A_{i}$ such that $a_{i} \backslash \hat{a}_{i} \neq \emptyset$. Then agent $i$ cannot announce $\tilde{s}_{i}^{a_{i}}$ with probability 1 because of the full-support assumption. Consequently, the expected transfer from agent $i$ would be strictly higher than the expected transfer under the equilibrium action $a_{i}$. To see this, observe that for any $s_{i}^{a_{i}} \in S_{i}^{a_{i}}$ announced by agent $i$ given action $\hat{a}_{i}$ and signal realization $\tilde{s}_{i}^{\hat{a}_{i}}=s_{i}^{\hat{a}_{i}}$,

$$
\begin{aligned}
E\left[t_{i}\left(s_{i}^{a_{i}}, \tilde{s}_{-i}^{a_{-i}}\right) \mid \tilde{s}_{i}^{\hat{a}_{i}}=s_{i}^{\hat{a}_{i}}\right] & =E\left[E\left[t_{i}\left(s_{i}^{a_{i}}, \tilde{s}_{-i}^{a_{-i}}\right) \mid \tilde{s}_{i}^{\hat{a}_{i}}, \tilde{s}_{i}^{Z_{i} \backslash \hat{a}_{i}}\right] \mid \tilde{s}_{i}^{\hat{a}_{i}}=s_{i}^{\hat{a}_{i}}\right] \\
& =E\left[E\left[t_{i}\left(s_{i}^{a_{i}}, \tilde{s}_{-i}^{a_{-i}}\right) \mid \tilde{s}_{i}^{a_{i}}, \tilde{s}_{i}^{Z_{i} \backslash a_{i}}\right] \mid \tilde{s}_{i}^{\hat{a}_{i}}=s_{i}^{\hat{a}_{i}}\right] \\
& >E\left[E\left[t_{i}\left(\tilde{s}_{i}^{a_{i}}, \tilde{s}_{-i}^{a_{-i}}\right) \mid \tilde{s}_{i}^{a_{i}}, \tilde{s}_{i}^{Z_{i} \backslash a_{i}}\right] \mid \tilde{s}_{i}^{\hat{a}_{i}}=s_{i}^{\hat{a}_{i}}\right],
\end{aligned}
$$

where the inequality follows from $(7)$ and $\operatorname{Pr}\left[\tilde{s}_{i}^{a_{i}} \neq s_{i}^{a_{i}} \mid \tilde{s}_{i}^{\hat{a}_{i}}=s_{i}^{\hat{a}_{i}}\right]>0$. 
Using this, we can show that it is not profitable for agent $i$ to acquire any $\hat{a}_{i} \neq a_{i}$. Let $s_{i}^{a_{i}}\left(s_{i}^{\hat{a}_{i}}\right)$ be any reporting strategy by agent $i$ after deviating to $\hat{a}_{i}$. Taking the expectation of the above expression with respect to $i$ 's signal given $\hat{a}_{i}$, we obtain

$$
\begin{aligned}
E\left[E\left[t_{i}\left(s_{i}^{a_{i}}\left(\tilde{s}_{i}^{\hat{a}_{i}}\right), \tilde{s}_{-i}^{a_{-i}}\right) \mid \tilde{s}_{i}^{\hat{a}_{i}}\right]\right] & >E\left[E\left[t_{i}\left(\tilde{s}_{i}^{a_{i}}, \tilde{s}_{-i}^{a_{-i}}\right) \mid \tilde{s}_{i}^{a_{i}}, \tilde{s}_{i}^{Z_{i} \backslash a_{i}}\right]\right] \\
& =E\left[E\left[t_{i}\left(\tilde{s}_{i}^{a_{i}}, \tilde{s}_{-i}^{a_{-i}}\right) \mid \tilde{s}_{i}^{a_{i}}\right]\right]
\end{aligned}
$$

Hence, agent $i$ loses money from the semi-robust lottery in expectation by choosing $\hat{a}_{i}$ rather than $a_{i}$. Once again, we can choose $\lambda$ large enough so that this expected loss outweighs any gains in the mechanism or in the cost of information acquisition. Therefore this deviation is not profitable either.

Corollary 2 (EFfiCient Implementation.) Consider a mechanism design problem $\left(\Omega, S^{Z}, q, p, X, u, c\right)$. If for each agent $i$ there exist semi-robust lotteries given an efficient level of information acquisition $a^{*}$, then $\left(a^{*}, f_{a^{*}}^{*}\right)$ can be implemented.

\section{Remark 2}

(i) Proposition 1 and Proposition 3 are complementary. Correlated information is necessary for the existence of a semi-robust lottery, hence Proposition 3 does not apply to the independent private value case, but Proposition 1 does. On the other hand, there is a situation to which Proposition 3 is applicable, but Proposition 1 is not. For example, take Example 2 and suppose that $\tilde{s}_{i, 1}, i=\alpha, \beta$ is not available. That is, the only additional information that is available is the signal that reveals $V$. Then the assumption for Proposition 1 is violated given efficient information acquisition. ${ }^{20}$ However, it is easy to verify that there exists a semi-robust lottery given efficient information acquisition in this example because $i$ 's additional signal about $V$ is not informative about $\tilde{s}_{j, 0}$.

(ii) We can directly check that there is no semi-robust lottery given efficient information acquisition in Example 2. For a semi-robust lottery, an agent must strictly prefer to announce $\omega_{\alpha}$ (resp. $\left.\omega_{\beta}\right)$ when he believes that the true state is more likely to be $\omega_{\alpha}$ (resp. $\left.\omega_{\beta}\right)$. But at the same time he must strictly prefer to announce $\omega_{\alpha}$ when $s_{i, 0}=\omega_{\alpha}$ and $s_{i, 1}=\omega_{\beta}$, i.e. he is sure that the state is $\omega_{\beta}$. This is clearly inconsistent.

(iii) Consider a standard mechanism design problem without information acquisition. In this environment, Condition B of d'Aspremont and Gérard-Varet [11] is necessary and sufficient for budget-balanced implementation of every social choice function:

Condition B: $\quad \exists t: S \rightarrow \mathbb{R}^{n}$ s.t. $\quad \sum_{i \in N} t_{i}(s)=0, \quad \forall s$

20 Note that $\left|S_{i}^{Z_{i}}\right|=|\Omega|=4>2=\left|S_{-i}^{a_{-i}^{*}}\right|$. 


$$
\sum_{s_{-i}} t_{i}\left(s_{i}, s_{-i}\right) h_{i}\left(s_{-i} \mid s_{i}\right)<\sum_{s_{-i}} t_{i}\left(\widehat{s}_{i}, s_{-i}\right) h_{i}\left(s_{-i} \mid s_{i}\right), \quad \forall s_{i}, \widehat{s}_{i} \neq s_{i}
$$

It is well known that the second part of Condition B is necessary and sufficient for implementation of every social choice function without budget balance. It implies that $t_{i}$ is a semi-robust lottery for agent $i$ in an environment without information acquisition and is equivalent to assuming that all conditional distributions are distinct, i.e. $h_{i}\left(\cdot \mid s_{i}\right) \neq h_{i}\left(\cdot \mid \widehat{s}_{i}\right)$ for any $s_{i}, \widehat{s}_{i} \neq s_{i}$ (see Aoyagi [1]).

The inequality in condition B is similar to our condition (7), but not directly applicable to our setting with information acquisition. Introduce information acquisition and suppose that $a=\left(a_{i}, a_{-i}\right)$ is chosen, with $a_{i} \subseteq Z_{i}$. Then the corresponding condition is that $h_{i}^{a_{-i}}\left(\cdot \mid s_{i}^{a_{i}}\right)$ are different for different $s_{i}^{a_{i}}$. If that is the case, then we can find a transfer $t_{i}$ that satisfies

$$
\sum_{s_{-i}^{a_{-i}}} t_{i}\left(s_{i}^{a_{i}}, s_{-i}^{a_{-i}}\right) h_{i}^{a_{-i}}\left(s_{-i}^{a_{-i}} \mid s_{i}^{a_{i}}\right)<\sum_{\substack{a_{-i} \\ s_{-i}}} t_{i}\left(\widehat{s}_{i}^{a_{i}}, s_{-i}^{a_{-i}}\right) h_{i}^{a_{-i}}\left(s_{-i}^{a_{-i}} \mid s_{i}^{a_{i}}\right)
$$

But this inequality is not enough for efficient implementation with information acquisition because agent $i$ 's belief conditional on $\left(s_{i}^{a_{i}}, s_{i}^{Z_{i} \backslash a_{i}}\right)$ can be close to $h_{i}^{a_{-i}}\left(\cdot \mid \widehat{s}_{i}^{a_{i}}\right)$ for some $\widehat{s}_{i}^{a_{i}} \neq s_{i}^{a_{i}}$ rather than $h_{i}^{a_{-i}}\left(\cdot \mid s_{i}^{a_{i}}\right)$ for some $s_{i} Z_{i} \backslash a_{i}$. Then agent $i$ may benefit from acquiring information and reporting $\widehat{s}_{i}^{a_{i}}$ instead of $s_{i}^{a_{i}}$ given additional information. ${ }^{21}$ Thus we need to strengthen this condition to (7) to guarantee efficient implementation with information acquisition.

(iv) In Bikhchandani [6], payment schemes called partially-robust lotteries were used for efficient implementation. A semi-robust lottery is a partiallyrobust lottery but not vice versa. A more restrictive payment scheme is needed in this paper in part because, unlike in [6], the information opportunities available to an agent cannot be ranked by informativeness.

In our setting with information acquisition, it may not be immediately clear whether there exist semi-robust lotteries for each agent. Below we provide a sufficient condition for the existence of semi-robust lotteries, which is easy to verify.

Fix $a \in A$. Let $\widehat{S}_{i}^{a_{i}}$ be any subset of $S_{i}^{a_{i}}$. We say that $s_{i}^{a_{i}} \in \widehat{S}_{i}^{a_{i}}$ can be separated from $\widehat{S}_{i}^{a_{i}}$ given $a$ if $h_{i}^{a_{-i}}\left(\cdot \mid s_{i}^{a_{i}}\right)$ can be represented by a convex combination of $h_{i}^{a_{-i}}\left(\cdot \mid \widehat{s}_{i}^{a_{i}}, s_{i}^{Z_{i} \backslash a_{i}}\right), \widehat{s}_{i}^{a_{i}} \in \widehat{S}_{i}^{a_{i}}, s_{i}^{Z_{i} \backslash a_{i}} \in S_{i}^{Z_{i} \backslash a_{i}}$ only by placing zero weight on $h_{i}^{a_{-i}}\left(\cdot \mid \widehat{s}_{i}^{a_{i}}, s_{i}^{Z_{i} \backslash a_{i}}\right)$ for any $\widehat{s}_{i}^{a_{i}} \neq s_{i}^{a_{i}}$ and $s_{i}^{Z_{i} \backslash a_{i}}$.

21 This suggests that information acquisition does not introduce any additional constraint when implementing the most informative action $a_{i}=Z_{i}$. This is in fact the case. If $h_{i}\left(\cdot \mid s_{i}^{Z_{i}}\right) \neq h_{i}\left(\cdot \mid \widehat{s}_{i}^{Z_{i}}\right)$ for any $s_{i}^{Z_{i}}, \widehat{s}_{i}^{Z_{i}} \neq s_{i}^{Z_{i}} \in S_{i}^{Z_{i}}$ for every $i$, then there exist semirobust lotteries given $a=\left(Z_{1}, \ldots, Z_{n}\right)$ for every $i$. Hence, any efficient allocation (in fact any allocation) is implementable in this case. 
Suppose that, given $a$, we can order $S_{i}^{a_{i}}$ as $s_{i}(1), s_{i}(2), \ldots$ in such a way that we can sequentially separate them one by one after eliminating the preceding elements, i.e., $s_{i}(1)$ is separated from $S_{i}^{a_{i}}, s_{i}(2)$ is separated from $S_{i}^{a_{i}} \backslash\left\{s_{i}(1)\right\}$ and so on. Then, as shown next, there exist semi-robust lotteries for agent $i$ given $a$.

Proposition 4 Suppose that, given action profile $a \in A, S_{i}^{a_{i}}$ can be ordered as $s_{i}(1), \ldots, s_{i}(R)$, where $R=\left|S_{i}^{a_{i}}\right|$, so that $s_{i}(r)$ is separated from $\left\{s_{i}(r+1), \ldots, s_{i}(R)\right\}$ for each $r=1,2, \ldots, R-1$. Then there exist semi-robust lotteries for agent $i$ given a.

The proof of Proposition 4 is in the appendix.

If there is no information acquisition, then our sequential separability condition is weaker than Crémer and McLean [9]'s necessary and sufficient condition for full surplus extraction. In our terminology, Crémer and McLean's condition -- that the belief of any type of an agent should not be a convex combination of other types of that agent -- is equivalent to saying that every $s_{i}^{a_{i}} \in S_{i}^{a_{i}}$ can be separated from $S_{i}^{a_{i}}$ simultaneously. On the other hand, we only require that agent $i$ 's signals can be separated sequentially in some order.

\section{Full Surplus Extraction}

Full surplus extraction occurs in a mechanism design problem if it is Bayesian incentive compatible and interim individually rational for agents to acquire the ex ante efficient information level and truthfully report their signals while the mechanism designer implements the ex post efficient rule and collects transfers such that each agent's interim expected utility is always zero. We need to strengthen the definition of semi-robust lotteries of Section 4 to obtain full surplus extraction.

$$
\begin{aligned}
& \text { A function } \pi_{i}: S^{a} \rightarrow \mathbb{R} \text { is a robust lottery given }\left(a_{i}, a_{-i}\right) \text { for agent } i \text { if } \\
& \qquad \begin{array}{l}
\sum_{a_{-i}} \pi_{i}\left(s_{i}^{a_{i}}, s_{-i}^{a_{-i}}\right) h_{i}^{a_{-i}}\left(s_{-i}^{a_{-i}} \mid s_{i}^{a_{i}}, s_{i}^{Z_{i} \backslash a_{i}}\right)=0, \quad \forall s_{i}^{a_{i}} \in S_{i}^{a_{i}} \\
\sum_{s_{-i}^{a_{-i}}} \pi_{i}\left(\widehat{s}_{i}^{a_{i}}, s_{-i}^{a_{-i}}\right) h_{i}^{a_{-i}}\left(s_{-i}^{a_{-i}} \mid s_{i}^{a_{i}}, s_{i}^{Z_{i} \backslash a_{i}}\right)>0,
\end{array} \quad \forall \widehat{s}_{i}^{a_{i}} \in S_{i}^{a_{i}}, \widehat{s}_{i}^{a_{i}} \neq s_{i}^{a_{i}}
\end{aligned}
$$

Thus semi-robust lotteries $t_{i}$ are robust if the left-hand side of (7) equals zero.

A comparison of robust lotteries with Crémer-McLean full surplus extraction lotteries (see (4) for a definition) is useful. A robust lottery given $a$ is a Crémer-McLean lottery when agents are costlessly endowed with information level $a$ and cannot acquire additional information. If $a_{i}=Z_{i}$ in (8) and (9), then a Crémer-McLean lottery for agent $i$ at information acquisition level $\left(Z_{i}, a_{-i}\right)$ is a robust lottery given $\left(Z_{i}, a_{-i}\right)$ for agent $i$. If, instead, $a_{i} \subsetneq Z_{i}$ then a Crémer-McLean lottery at $\left(a_{i}, a_{-i}\right)$ need not be robust for agent $i$. As 
Example 1 shows, a robust lottery may not exist even though for generic beliefs the sets of Crémer-McLean lotteries at $\left(\hat{a}_{i}, a_{-i}\right)$ are non-empty for all $\hat{a}_{i}$ such that $a_{i} \subseteq \hat{a}_{i}$. A sufficient condition for the existence of robust lotteries is provided in Proposition 6.

Next, we show that the existence of robust lotteries is sufficient for full surplus extraction.

Proposition 5 Consider an information structure $\left(\Omega, S^{Z}, q, p\right)$ and an information acquisition decision $a=\left(a_{1}, a_{2}, \ldots, a_{n}\right)$. Suppose that for each agent $i$ there exist robust lotteries given a. Then for any mechanism design problem on this information structure and any social choice function $f$, the social choice rule $(a, f)$ can be implemented such that each agent's interim expected surplus is zero.

Proof: Let $f$ be a social choice function for a mechanism design problem on this information structure. Let $\pi_{i}$ be a robust lottery for agent $i$ given $a$. Define

$$
t_{i}\left(s^{a}\right)=\lambda \pi_{i}\left(s^{a}\right)+g_{i}\left(s^{a}\right), \quad \forall s^{a} \in S^{a},
$$

where $\lambda>0$ and

$$
g_{i}\left(s_{i}^{a_{i}}, s_{-i}^{a_{-i}}\right)=V_{i}\left(f_{i}\left(s_{i}^{a_{i}}, s_{-i}^{a_{-i}}\right),\left(s_{i}^{a_{i}}, s_{-i}^{a_{-i}}\right)\right)-c_{i}\left(a_{i}\right)
$$

It is straightforward to check that the mechanism $(a, f, t)$ satisfies $(3)$ with the left-hand side equal to $c_{i}\left(a_{i}\right)$.

By choosing $\lambda$ sufficiently large we can ensure that for all $s_{i}^{a_{i}} \in S_{i}^{a_{i}}$ and $\widehat{s}_{i}^{a_{i}} \neq s_{i}^{a_{i}}$,

$$
\begin{aligned}
& E\left[V_{i}\left(f\left(s_{i}^{a_{i}}, \tilde{s}_{-i}^{a_{-i}}\right),\left(s_{i}^{a_{i}}, \tilde{s}_{-i}^{a_{-i}}\right)\right)-t_{i}\left(s_{i}^{a_{i}}, \tilde{s}_{-i}^{a_{-i}}\right) \mid \tilde{s}_{i}^{a_{i}}=s_{i}^{a_{i}}\right] \\
= & E\left[V_{i}\left(f\left(s_{i}^{a_{i}}, \tilde{s}_{-i}^{a_{-i}}\right),\left(s_{i}^{a_{i}}, \tilde{s}_{-i}^{a_{-i}}\right)\right)-g_{i}\left(s_{i}^{a_{i}}, \tilde{s}_{-i}^{a_{-i}}\right) \mid \tilde{s}_{i}^{a_{i}}=s_{i}^{a_{i}}\right]-\lambda \sum_{s_{-i}} \pi_{i}\left(s_{i}^{a_{i}}, s_{-i}^{a_{-i}}\right) h_{i}^{a_{-i}}\left(s_{-i}^{a_{-i}} \mid s_{i}^{a_{i}}\right) \\
= & E\left[V_{i}\left(f\left(s_{i}^{a_{i}}, \tilde{s}_{-i}^{a_{-i}}\right),\left(s_{i}^{a_{i}}, \tilde{s}_{-i}^{a_{-i}}\right)\right)-g_{i}\left(s_{i}^{a_{i}}, \tilde{s}_{-i}^{a_{-i}}\right) \mid \tilde{s}_{i}^{a_{i}}=s_{i}^{a_{i}}\right] \\
> & E\left[V_{i}\left(f\left(\widehat{s}_{i}^{a_{i}}, \tilde{s}_{-i}^{a_{-i}}\right),\left(s_{i}^{a_{i}}, \tilde{s}_{-i}^{a_{-i}}\right)\right)-g_{i}\left(\widehat{s}_{i}^{a_{i}}, \tilde{s}_{-i}^{a_{-i}}\right) \mid \tilde{s}_{i}^{a_{i}}=s_{i}^{a_{i}}\right]-\lambda \sum_{s_{-i}} \pi_{i}\left(\widehat{s}_{i}^{a_{i}}, s_{-i}^{a_{-i}}\right) h_{i}^{a_{-i}}\left(s_{-i}^{a_{-i}} \mid s_{i}^{a_{i}}\right) \\
= & E\left[V_{i}\left(f\left(\widehat{s}_{i}^{a_{i}}, \tilde{s}_{-i}^{a_{-i}}\right),\left(s_{i}^{a_{i}}, \tilde{s}_{-i}^{a_{-i}}\right)\right)-t_{i}\left(\widehat{s}_{i}^{a_{i}}, \tilde{s}_{-i}^{a_{-i}}\right) \mid \tilde{s}_{i}^{a_{i}}=s_{i}^{a_{i}}\right],
\end{aligned}
$$

where the second equality follows from (8) and the inequality from (9). Thus, if agent $i$ chooses information level $a_{i}$, he has no incentive to lie or opt out after any realization of his signal.

Suppose, instead, that agent $i$ chooses information level $\hat{a}_{i} \supset a_{i}$. The use of robust lotteries ensures that, conditional on selection of $\hat{a}_{i} \supset a_{i}$, the optimal strategy is to truthfully announce $s_{i}^{a_{i}}$ when $s_{i}^{\hat{a}_{i}}=\left(s_{i}^{a_{i}}, s_{i}^{\hat{a}_{i} \backslash a_{i}}\right)$ is observed; announcing $\widehat{s}_{i}^{a_{i}} \neq s_{i}^{a_{i}}$ results in a large negative expected payoff. Without taking into account the cost of information acquisition, agent $i$ 's expected 
payoff after observing $\tilde{s}_{i}^{\hat{a}_{i}}=\left(\tilde{s}_{i}^{a_{i}}, \tilde{s}_{i}^{\hat{a}_{i} \backslash a_{i}}\right)$ would be the same as after observing $\tilde{s}_{i}^{a_{i}}$ :

$$
\begin{aligned}
& E\left[E\left[V_{i}\left(f\left(\tilde{s}_{i}^{a_{i}}, \tilde{s}_{-i}^{a_{-i}}\right),\left(\tilde{s}_{i}^{a_{i}}, \tilde{s}_{i}^{\hat{a}_{i} \backslash a_{i}}\right), \tilde{s}_{-i}^{a_{-i}}\right)-t_{i}\left(\tilde{s}_{i}^{a_{i}}, \tilde{s}_{-i}^{a_{-i}}\right) \mid \tilde{s}_{i}^{a_{i}}, \tilde{s}_{i}^{\hat{a}_{i} \backslash a_{i}}\right]\right] \\
= & E\left[E\left[V_{i}\left(f\left(\tilde{s}_{i}^{a_{i}}, \tilde{s}_{-i}^{a_{-i}}\right), \tilde{s}_{i}^{a_{i}}, \tilde{s}_{-i}^{a_{-i}}\right)-t_{i}\left(\tilde{s}_{i}^{a_{i}}, \tilde{s}_{-i}^{a_{-i}}\right) \mid \tilde{s}_{i}^{a_{i}}\right]\right],
\end{aligned}
$$

where the equality follows from (1) and (8). Agent $i$ would lose $c_{i}\left(\hat{a}_{i}\right)-c_{i}\left(a_{i}\right)>$ 0 by deviating to $\hat{a}_{i} \supset a_{i}$, making this deviation unprofitable.

Finally, suppose that agent $i$ chooses information level $\hat{a}_{i}$ such that $a_{i} \backslash \hat{a}_{i} \neq$ $\emptyset$. Then, an argument similar to that in the last paragraph of the proof of Proposition 3 implies that if agent $i$ announces any $s_{i}^{a_{i}} \in S_{i}^{a_{i}}$, then given any $\tilde{s}_{i}^{\hat{a}_{i}}=s_{i}^{\hat{a}_{i}}$,

$$
\begin{aligned}
E\left[\pi_{i}\left(s_{i}^{a_{i}}, \tilde{s}_{-i}^{a_{-i}}\right) \mid \tilde{s}_{i}^{\hat{a}_{i}}=s_{i}^{\hat{a}_{i}}\right] & =E\left[E\left[\pi_{i}\left(s_{i}^{a_{i}}, \tilde{s}_{-i}^{a_{-i}}\right) \mid \tilde{s}_{i}^{\hat{a}_{i}}, \tilde{s}_{i}^{Z_{i} \backslash \hat{a}_{i}}\right] \mid \tilde{s}_{i}^{\hat{a}_{i}}=s_{i}^{\hat{a}_{i}}\right] \\
& =E\left[E\left[\pi_{i}\left(s_{i}^{a_{i}}, \tilde{s}_{-i}^{a_{-i}}\right) \mid \tilde{s}_{i}^{a_{i}}, \tilde{s}_{i}^{Z_{i} \backslash a_{i}}\right] \mid \tilde{s}_{i}^{\hat{a}_{i}}=s_{i}^{\hat{a}_{i}}\right] \\
& >E\left[\left[\pi_{i}\left(\tilde{s}_{i}^{a_{i}}, \tilde{s}_{-i}^{a_{-i}}\right) \mid \tilde{s}_{i}^{a_{i}}, \tilde{s}_{i}^{Z_{i} \backslash a_{i}}\right] \mid \tilde{s}_{i}^{\hat{a}_{i}}=s_{i}^{\hat{a}_{i}}\right] \\
& =0
\end{aligned}
$$

where the inequality follows from (9) and $\operatorname{Pr}\left[\tilde{s}_{i}^{a_{i}} \neq s_{i}^{a_{i}} \mid \tilde{s}_{i}^{\hat{a}_{i}}=s_{i}^{\hat{a}_{i}}\right]>0$. Hence it is not profitable to deviate from the prescribed level of information acquisition as shown in the proof of Proposition 3.

By choosing $\lambda>0$ sufficiently large one can ensure that after choosing $\hat{a}_{i}$, agent $i$ is better off not participating, thus yielding a payoff of $-c_{i}\left(\hat{a}_{i}\right)$, rather than participate and lie about observing some $s_{i}^{a_{i}}$ which yields a payoff smaller than $-c_{i}\left(\hat{a}_{i}\right)$. Hence, it is not profitable to choose $\hat{a}_{i}$ rather than $a_{i}$.

Recall that $a^{*}$ is the ex ante efficient information acquisition level and $f_{a^{*}}^{*}$ is the ex post efficient rule associated with a mechanism design problem. The following is immediate.

Corollary 3 (Full surplus EXTRACTION.) Consider a mechanism design problem $\left(\Omega, S^{Z}, q, p, X, u, c\right)$. If for each agent $i$ there exist robust lotteries given an efficient level of information acquisition $a^{*}$, then there exists a mechanism $\left(a^{*}, f_{a^{*}}^{*}, t^{*}\right)$ that is incentive compatible, interim individually rational, and satisfies

$$
t_{i}^{*}\left(s_{i}^{a_{i}^{*}}, s_{-i}^{a_{-i}^{*}}\right)=V_{i}\left(f_{a^{*}}^{*}\left(s_{i}^{a_{i}^{*}}, s_{-i}^{a_{-i}^{*}}\right),\left(s_{i}^{a_{i}^{*}}, s_{-i}^{a_{-i}^{*}}\right)\right)-c_{i}\left(a_{i}^{*}\right) .
$$

for any $\left(s_{i}^{a_{i}^{*}}, s_{-i}^{a_{-i}^{*}}\right) \in S^{a^{*}}$ for any $i \in N$. 
We end this section with a characterization result for robust lotteries. The necessary and sufficient condition in Proposition A is stronger than the sufficient condition of Proposition 4.

Proposition A (BikhChandani [6]). Robust lotteries given $\left(a_{i}, a_{-i}\right)$ exist for agent $i$ iff for each $s_{i}^{a_{i}} \in S_{i}^{a_{i}}$, the set of linear combinations of beliefs over $S_{-i}^{a_{-i}}, h_{i}^{a_{-i}}\left(\cdot \mid s_{i}^{a_{i}}, s_{i}^{Z_{i} \backslash a_{i}}\right), s_{i}^{Z_{i} \backslash a_{i}} \in S_{i}^{Z_{i} \backslash a_{i}}$, does not intersect with the convex hull of beliefs $h_{i}^{a_{-i}}\left(\cdot \mid \widehat{s}_{i}^{Z_{i}}\right), \forall \widehat{s}_{i}^{Z_{i}}=\left(\widehat{s}_{i}^{a_{i}}, \widehat{s}_{i}^{Z_{i} \backslash a_{i}}\right) \in S_{i}^{Z_{i}}$ such that $\widehat{s}_{i}^{a_{i}} \neq s_{i}^{a_{i}}$.

This leads to a sufficient condition for generic full surplus extraction in terms of the cardinality of the sets of signals and states of nature.

Proposition 6 Fix $\left(\Omega, S^{Z}\right)$. If $\left|S_{i}^{Z_{i}}\right| \leq \min \left\{|\Omega|,\left|S_{-i}^{\mathbf{0}}\right|\right\}$ for each $i$, then full surplus extraction is possible for any mechanism design problem $\left(\Omega, S^{Z}, q, p, X, u, c\right)$ for an open and dense set of $(q, p)$.

Proof: Proposition A implies that full surplus extraction is possible given $\left(a_{i}, a_{-i}\right)$ if $h_{i}^{a_{-i}}\left(\cdot \mid s_{i}^{Z_{i}}\right), s_{i}^{Z_{i}} \in S_{i}^{Z_{i}}$ are linearly independent. This is satisfied if $h_{i}^{\mathbf{0}}\left(\cdot \mid s_{i}^{Z_{i}}\right), s_{i}^{Z_{i}} \in S_{i}^{Z_{i}}$ are linearly independent. As in the proof of Proposition 2, we can show that this is the case for a generic choice of $(q, p)$ if $\left|S_{i}^{Z_{i}}\right| \leq$ $\min \left\{|\Omega|,\left|S_{-i}^{\mathbf{0}}\right|\right\}$ for each $i$.

In Example 1, the necessary and sufficient condition of Proposition A is not satisfied. After agent $\alpha$ acquires perfect information about $\tilde{\omega}$, his beliefs over $\tilde{s}_{\beta, 0}$ are the same whether $\tilde{s}_{\alpha, 0}=\ell$ or $h$.

If, instead of assuming that agents' signals are distributed independently conditional of $\omega$, we assume that $\left(\tilde{w}, \tilde{s}_{i}^{Z_{i}}, \tilde{s}_{-i}^{Z_{-i}}\right)$ are jointly distributed, then the hypothesis of Proposition 6 can be weakened to $\left|S_{i}^{Z_{i}}\right| \leq\left|S_{-i}^{0}\right|$.

\section{Discussion}

This paper examines when efficient implementation and full surplus extraction are Bayesian incentive compatible and interim individually rational in a setting with covert information acquisition by agents. What makes this problem nontrivial is that we need to implement both efficient allocation and efficient information acquisition. We illustrate by an example that correlation of agents' private signals does not guarantee even efficient implementation.

It is interesting to compare sufficient conditions for efficient implementation and for full surplus extraction. First, as already noted, the sufficient conditions for (the existence of) semi-robust lotteries in Proposition 4 are weaker than the necessary and sufficient conditions for robust lotteries in Proposition A. Second, Proposition 2 shows that the sufficient condition for efficient implementation in Proposition 1 is generically satisfied if

$$
\left|S_{-i}^{\mathbf{0}}\right| \geq \min \left\{|\Omega|,\left|S_{i}^{Z_{i}}\right|\right\}
$$


Proposition 6 establishes that a generic sufficient condition for existence of robust lotteries is

$$
\left|S_{i}^{Z_{i}}\right| \leq \min \left\{|\Omega|,\left|S_{-i}^{\mathbf{0}}\right|\right\}
$$

If (11) is satisfied then so is (10). It follows from (11) that $\min \left\{|\Omega|,\left|S_{i}^{Z_{i}}\right|\right\}$ is less than or equal to $\min \left\{|\Omega|,\left|S_{-i}^{\mathbf{0}}\right|\right\}$, which is less than or equal to $\left|S_{-i}^{\mathbf{0}}\right|$. Not surprisingly, the sufficient conditions for efficient implementation are weaker than those for full surplus extraction.

We have not pursued the tightness of these conditions. Identifying necessary and sufficient conditions for efficient implementation and full surplus extraction are left for future research.

We can obtain similar results under much weaker assumptions on the information structure if we relax exact efficiency/FSE to approximate efficiency/FSE and allow the mechanism to implement random action profiles. Each agent's incentive constraints and interim individually rational constraints are more easily satisfied when the other agents' signals are more informative, i.e., the other agents acquire more information. For exact efficiency, the level of information acquisition must be at the efficient level. For approximate efficiency, however, we can dispense with this upper bound of information by letting agents acquire the maximum amount of information with small probability. In this way, we can exploit the maximum amount of information with very little loss in efficiency. ${ }^{22}$

We noted in Remark 1(iv) that Proposition 1 extends to sequential information within agents. One can also have sequential information acquisition across agents, where agents observe their signals sequentially and report them to the mechanism designer who makes information-acquisition decisions across agents using realizations of all the signals that have been previously observed by the agents. ${ }^{23}$ It would be interesting to examine sequential information acquisition across agents in this model.

\footnotetext{
22 A similar exercise is done in Obara [24] and Rahman and Obara [26]. Some results in Fudenberg, Levine and Maskin [14] and Kandori [20] are based on a similar idea.

23 Gershkov and Szentes [15] characterize the optimal voting mechanism with sequential information acquisition without monetary transfer when agents have identical preferences.
} 


\section{Appendix: Omitted Proofs}

Proof of Corollary 1: We show that the condition for the corollary implies the condition for Proposition 1. This condition means that the matrix $D_{i} P_{-i}$ has rank $\left|S_{i}^{Z_{i}}\right|$ (i.e. full row rank), where $D_{i}$ and $P_{-i}$ are as defined in the proof of Proposition 1. Then we can obtain a full column rank matrix $P_{-i}^{\prime}$ by selecting independent $\left|S_{i}^{Z_{i}}\right|$ columns from $P_{-i}$ such that $D_{i} P_{-i}^{\prime}$ is a regular matrix. Now we show that $M^{Z_{i}}$ and the columns of $P_{-i}^{\prime}$ forms a basis of $\mathbb{R}^{\left|\Omega_{-i}\right|}$. Suppose not. Note that the number of columns for $P_{-i}^{\prime}$ is exactly $\left|S_{i}^{Z_{i}}\right|$ and the number of vectors in $M^{Z_{i}}$ is $\left|\Omega_{-i}\right|-\left|S_{i}^{Z_{i}}\right|$. Hence all those vectors in $\mathbb{R}^{\left|\Omega_{-i}\right|}$ cannot be independent. Then there must exist a nonzero vector $x \in \mathbb{R}^{\left|S_{-i}^{a_{i}}\right|}$ such that $P_{-i}^{\prime} x$ is in the null space of $D_{i}$, which implies $D_{i} P_{-i}^{\prime} x=0$. This is a contradiction to $D_{i} P_{-i}^{\prime}$ being regular.

To prove Proposition 4, we first prove a lemma. In the following, we fix an action profile $a \in A$ and denote $h_{i}^{a_{-i}}$ by $h_{i}$ to simplify the exposition.

Consider the following condition on the information structure.

Condition 1: If $\lambda: S_{i}^{a_{i}} \times S_{i}^{a_{i}} \times S_{i}^{Z_{i} \backslash a_{i}} \rightarrow \mathbb{R}_{+}$satisfies

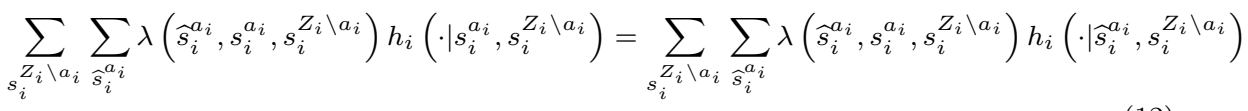

and

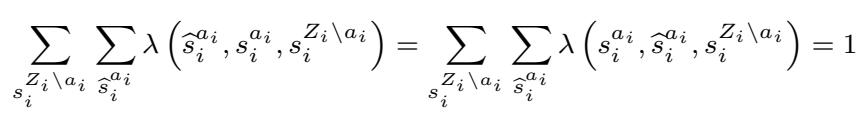

for any $s_{i}^{a_{i}}$, then $\lambda\left(s_{i}^{a_{i}}, \widehat{s}_{i}^{a_{i}}, s_{i}^{Z_{i} \backslash a_{i}}\right)$ must be 0 for any $s_{i}^{a_{i}}, \widehat{s}_{i}^{a_{i}} \neq s_{i}^{a_{i}}$ and $s_{i}^{Z_{i} \backslash a_{i}}$.

The following lemma shows that this condition is equivalent to the existence of a semi-robust lottery for every $s_{i}^{a_{i}} \in S_{i}^{a_{i}}$.

Lemma 1 Condition 1 is satisfied if and only if there exist semi-robust lotteries given a, i.e., there exists $t_{i}: S_{i}^{a_{i}} \times S_{-i}^{a_{-i}} \rightarrow \mathbb{R}$ that satisfies

$$
\sum_{s_{-i}^{a_{-i}}} t_{i}\left(s_{i}^{a_{i}}, s_{-i}^{a_{-i}}\right) h_{i}\left(s_{-i}^{a_{-i}} \mid s_{i}^{a_{i}}, s_{i}^{Z_{i} \backslash a_{i}}\right)<\sum_{s_{-i}^{a_{-i}}} t_{i}\left(\widehat{s}_{i}^{a_{i}}, s_{-i}^{a_{-i}}\right) h_{i}\left(s_{-i}^{a_{-i}} \mid s_{i}^{a_{i}}, s_{i}^{Z_{i} \backslash a_{i}}\right)
$$

for any $s_{i}^{a_{i}}, \widehat{s}_{i}^{a_{i}} \neq s_{i}^{a_{i}} \in S_{i}^{a_{i}}$ and for any $s_{i}^{Z_{i} \backslash a_{i}} \in S_{i}^{Z_{i} \backslash a_{i}}$.

Proof: There exists such $t_{i}: S_{i}^{a_{i}} \times S_{-i}^{a_{-i}} \rightarrow \mathbb{R}$ if and only if the LP below has a feasible solution:

$$
\min _{t} 0
$$


s.t.

$$
\begin{gathered}
\sum_{\substack{a_{-i} \\
s_{-i}}} t\left(\widehat{s}_{i}^{a_{i}}, s_{-i}^{a_{-i}}\right) h_{i}\left(s_{-i}^{a_{-i}} \mid s_{i}^{a_{i}}, s_{i}^{Z_{i} \backslash a_{i}}\right)-\sum_{\substack{a_{-i} \\
s_{-i}}} t\left(s_{i}^{a_{i}}, s_{-i}^{a_{-i}}\right) h_{i}\left(s_{-i}^{a_{-i}} \mid s_{i}^{a_{i}}, s_{i}^{Z_{i} \backslash a_{i}}\right) \geq 1 \\
\forall s_{i}^{Z_{i} \backslash a_{i}}, \forall s_{i}^{a_{i}}, \forall \widehat{s}_{i}^{a_{i}} \neq s_{i}^{a_{i}} .
\end{gathered}
$$

Its dual is

$$
\max _{\lambda \geq 0} \sum_{s_{i}^{a_{i}}} \sum_{\widehat{s}_{i}^{a_{i}} \neq s_{i}^{a_{i}}} \sum_{s_{i}^{Z_{i} \backslash a_{i}}} \lambda\left(\widehat{s}_{i}^{a_{i}}, s_{i}^{a_{i}}, s_{i}^{Z_{i} \backslash a_{i}}\right)
$$

s.t.

$$
\begin{gathered}
\sum_{s_{i}^{Z_{i} \backslash a_{i}}} \sum_{\widehat{s}_{i}^{a_{i}} \neq s_{i}^{a_{i}}} \lambda\left(\widehat{s}_{i}^{a_{i}}, s_{i}^{a_{i}}, s_{i}^{Z_{i} \backslash a_{i}}\right) h_{i}\left(s_{-i}^{a_{-i}} \mid s_{i}^{a_{i}}, s_{i}^{Z_{i} \backslash a_{i}}\right) \\
=\sum_{\substack{Z_{i} \backslash a_{i} \\
s_{i} a_{i}^{a_{i}} \neq s_{i}^{a_{i}}}} \lambda\left(s_{i}^{a_{i}}, \widehat{s}_{i}^{a_{i}}, s_{i}^{Z_{i} \backslash a_{i}}\right) h_{i}\left(s_{-i}^{a_{-i}} \mid \widehat{s}_{i}^{a_{i}}, s_{i}^{Z_{i} \backslash a_{i}}\right), \\
\forall s_{i}^{a_{i}}, \forall s_{-i}^{a_{-i}}
\end{gathered}
$$

The LP has a feasible solution iff every feasible solution to the dual satisfies $\lambda\left(\widehat{s}_{i}^{a_{i}}, s_{i}^{a_{i}}, s_{i}^{Z_{i} \backslash a_{i}}\right)=0$ for all $s_{i}^{a_{i}}$, for all $\widehat{s}_{i}^{a_{i}} \neq s_{i}^{a_{i}}$, for all $s_{i}^{Z_{i} \backslash a_{i}}$. Summing (14) over $s_{-i}^{a_{-i}}$ we have

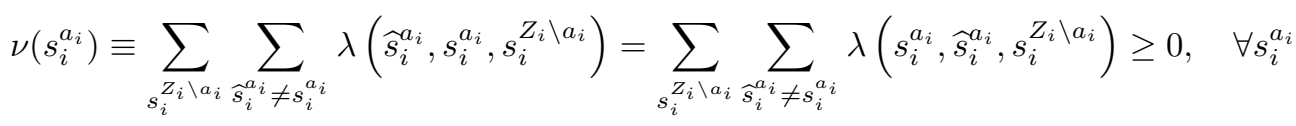

Let $B$ be any large enough number so that $B>\nu\left(s_{i}^{a_{i}}\right)$ for all $s_{i}^{a_{i}}$ and select any $\lambda\left(s_{i}^{a_{i}}, s_{i}^{a_{i}}, s_{i}^{Z_{i} \backslash a_{i}}\right) \geq 0$ for each $s_{i}^{a_{i}}$ so that

$$
\sum_{\substack{Z_{i} \backslash a_{i} \\ s_{i}}} \lambda\left(s_{i}^{a_{i}}, s_{i}^{a_{i}}, s_{i}^{Z_{i} \backslash a_{i}}\right)=B-\nu\left(s_{i}^{a_{i}}\right)>0
$$

Then we have

$$
\sum_{s_{i}^{Z_{i} \backslash a_{i}}{ }_{\widehat{s}_{i}}} \sum_{\hat{s}_{i}} \lambda\left(\widehat{s}_{i}^{a_{i}}, s_{i}^{a_{i}}, s_{i}^{Z_{i} \backslash a_{i}}\right)=\sum_{s_{i}^{Z_{i} \backslash a_{i}}{ }_{\widehat{s}_{i}}} \sum_{a_{i}} \lambda\left(s_{i}^{a_{i}}, \widehat{s}_{i}^{a_{i}}, s_{i}^{Z_{i} \backslash a_{i}}\right)=B>0
$$

Then (14) is equivalent to

$$
\begin{gathered}
\sum_{s_{i}^{Z_{i} \backslash a_{i}}} \sum_{\widehat{s}_{i}^{a_{i}}} \lambda\left(\widehat{s}_{i}^{a_{i}}, s_{i}^{a_{i}}, s_{i}^{Z_{i} \backslash a_{i}}\right) h_{i}\left(s_{-i}^{a_{-i}} \mid s_{i}^{a_{i}}, s_{i}^{Z_{i} \backslash a_{i}}\right) \\
=\sum_{s_{i}^{Z_{i} \backslash a_{i}}} \sum_{\widehat{s}_{i}^{a_{i}}} \lambda\left(s_{i}^{a_{i}}, \widehat{s}_{i}^{a_{i}}, s_{i}^{Z_{i} \backslash a_{i}}\right) h_{i}\left(s_{-i}^{a_{-i}} \mid \widehat{s}_{i}^{a_{i}}, s_{i}^{Z_{i} \backslash a_{i}}\right), \\
\forall s_{i}^{a_{i}}, \forall s_{-i}^{a_{-i}},
\end{gathered}
$$

which is (12). Without loss of generality, let $B=1$ and the lemma follows. 
Proof of Proposition 4: We just need to show that the assumption on $S_{i}^{a_{i}}$ for Proposition 4 implies Condition 1. Suppose that $S_{i}^{a_{i}}$ can be ordered as assumed and that $\lambda \geq 0$ satisfies (12) and (13). Then the following equation holds for $s_{i}(1) \in S_{i}^{a_{i}}$ :

$$
\begin{aligned}
& \sum_{s_{i} \backslash a_{i}} \sum_{\widehat{s}_{i}^{a_{i}}} \lambda\left(\widehat{s}_{i}^{a_{i}}, s_{i}(1), s_{i}^{Z_{i} \backslash a_{i}}\right) h_{i}\left(\cdot \mid s_{i}(1), s_{i}^{Z_{i} \backslash a_{i}}\right) \\
= & \sum_{s_{i}^{Z_{i} \backslash a_{i}}} \sum_{\widehat{s}_{i}^{a_{i}}} \lambda\left(s_{i}(1), \widehat{s}_{i}^{a_{i}}, s_{i}^{Z_{i} \backslash a_{i}}\right) h_{i}\left(\cdot \mid \widehat{s}_{i}^{a_{i}}, s_{i}^{Z_{i} \backslash a_{i}}\right)
\end{aligned}
$$

We can define $g\left(s_{i}^{a_{i}}(1), s_{i}^{Z_{i} \backslash a_{i}}\right)$ and $\eta>0$ so that the following equation is satisfied for every $s_{i}^{Z_{i} \backslash a_{i}}$ :

$$
\frac{g\left(s_{i}(1), s_{i}^{Z_{i} \backslash a_{i}}\right)+\sum_{\widehat{s}_{i} a_{i}} \lambda\left(\widehat{s}_{i}^{a_{i}}, s_{i}(1), s_{i}^{Z_{i} \backslash a_{i}}\right)}{\eta}=\operatorname{Pr}\left(\widetilde{s}_{i}^{Z_{i} \backslash a_{i}}=s_{i}^{Z_{i} \backslash a_{i}} \mid s_{i}(1)\right)
$$

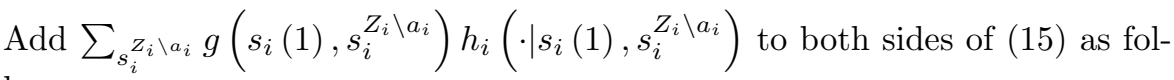
lows:

$$
\begin{aligned}
& \sum_{s_{i} \backslash a_{i}}\left\{\sum_{\hat{s}_{i}^{a}} \lambda\left(\widehat{s}_{i}^{a_{i}}, s_{i}(1), s_{i}^{Z_{i} \backslash a_{i}}\right)+g\left(s_{i}(1), s_{i}^{Z_{i} \backslash a_{i}}\right)\right\} h_{i}\left(\cdot \mid s_{i}(1), s_{i}^{Z_{i} \backslash a_{i}}\right) \\
= & \sum_{s_{i} \backslash a_{i}} \sum_{\widehat{s}_{i}^{a_{i}} \neq s_{i}(1)} \lambda\left(s_{i}(1), \widehat{s}_{i}^{a_{i}}, s_{i}^{Z_{i} \backslash a_{i}}\right) h_{i}\left(\cdot \mid \widehat{s}_{i}^{a_{i}}, s_{i}^{Z_{i} \backslash a_{i}}\right) \\
& +\sum_{s_{i}^{Z_{i} \backslash a_{i}}}\left\{\lambda\left(s_{i}(1), s_{i}(1), s_{i}^{Z_{i} \backslash a_{i}}\right)+g\left(s_{i}(1), s_{i}^{Z_{i} \backslash a_{i}}\right)\right\} h_{i}\left(\cdot \mid s_{i}(1), s_{i}^{Z_{i} \backslash a_{i}}\right)
\end{aligned}
$$

Divide both sides by $\eta$. Then we obtain

$$
\begin{aligned}
& \sum_{s_{i} \backslash a_{i}} \operatorname{Pr}\left(\widetilde{s}_{i}^{Z Z_{i} \backslash a_{i}}=s_{i}^{Z_{i} \backslash a_{i}} \mid s_{i}(1)\right) h_{i}\left(\cdot \mid s_{i}(1), s_{i}^{Z_{i} \backslash a_{i}}\right) \\
= & h_{i}\left(\cdot \mid s_{i}(1)\right) \\
= & \frac{1}{\eta} \sum_{s_{i}^{Z_{i} \backslash a_{i}}{ }_{\widehat{s}_{i}} \sum_{i} \neq s_{i}(1)} \lambda\left(s_{i}(1), \widehat{s}_{i}^{a_{i}}, s_{i}^{Z_{i} \backslash a_{i}}\right) h_{i}\left(\cdot \mid \widehat{s}_{i}^{a_{i}}, s_{i}^{Z_{i} \backslash a_{i}}\right) \\
& +\frac{1}{\eta} \sum_{s_{i}^{Z_{i} \backslash a_{i}}}\left\{\lambda\left(s_{i}(1), s_{i}(1), s_{i}^{Z_{i} \backslash a_{i}}\right)+g\left(s_{i}(1), s_{i}^{Z_{i} \backslash a_{i}}\right)\right\} h_{i}\left(\cdot \mid s_{i}(1), s_{i}^{Z_{i} \backslash a_{i}}\right)
\end{aligned}
$$

Since $s_{i}(1)$ is separated from $S_{i}^{a_{i}}$ by assumption, this implies $\lambda\left(s_{i}(1), \widehat{s}_{i}^{a_{i}}, s_{i}^{Z_{i} \backslash a_{i}}\right)=$ 0 for any $\widehat{s}_{i}^{a_{i}} \neq s_{i}(1)$ and $s_{i}^{Z_{i} \backslash a_{i}}$. 
Note that $\sum_{s_{i}^{Z_{i} \backslash a_{i}}} \lambda\left(s_{i}(1), s_{i}(1), s_{i}^{Z_{i} \backslash a_{i}}\right)=1$ by (13). This in turn implies $\lambda\left(\widehat{s}_{i}^{a_{i}}, s_{i}(1), s_{i}^{Z_{i} \backslash a_{i}}\right)=0$ for any $\widehat{s}_{i}^{a_{i}} \neq s_{i}(1)$ and $s_{i}^{Z_{i} \backslash a_{i}}$ by (13) again.

The rest of the proof is by induction. Suppose that for every $r=1,2, \ldots, m$, $\lambda\left(s_{i}(r), \widehat{s}_{i}^{a_{i}}, s_{i}^{Z_{i} \backslash a_{i}}\right)=\lambda\left(\widehat{s}_{i}^{a_{i}}, s_{i}(r), s_{i}^{Z_{i} \backslash a_{i}}\right)=0$ for any $\widehat{s}_{i}^{a_{i}} \neq s_{i}(r)$ and $s_{i}^{Z_{i} \backslash a_{i}}$ by (13). For $s_{i}(m+1)$, we get

$$
\begin{aligned}
& \sum_{s_{i}^{Z_{i} \backslash a_{i}}} \sum_{\hat{s}_{i}^{a_{i}} \in S_{i}^{a_{i}} \backslash\left\{s_{i}(1), \ldots, s_{i}(m)\right\}} \lambda\left(\widehat{s}_{i}^{a_{i}}, s_{i}(m+1), s_{i}^{Z_{i} \backslash a_{i}}\right) h_{i}\left(\cdot \mid s_{i}(m+1), s_{i}^{Z_{i} \backslash a_{i}}\right) \\
& =\sum_{s_{i}^{Z_{i} \backslash a_{i}}{ }_{\widehat{s}_{i} a_{i} \in S_{i}^{a_{i}} \backslash\left\{s_{i}(1), \ldots, s_{i}(m)\right\}}} \lambda\left(s_{i}(m+1), \widehat{s}_{i}^{a_{i}}, s_{i}^{Z_{i} \backslash a_{i}}\right) h_{i}\left(\cdot \mid \widehat{s}_{i}^{a_{i}}, s_{i}^{Z_{i} \backslash a_{i}}\right)
\end{aligned}
$$

As in the first step, define $g\left(s_{i}(m+1), s_{i}^{Z_{i} \backslash a_{i}}\right)$ and $\eta>0$ that satisfies

$$
\begin{array}{r}
g\left(s_{i}(m+1), s_{i}^{Z_{i} \backslash a_{i}}\right)+\sum_{\widehat{s}_{i} a_{i} \in S_{i}^{a_{i}} \backslash\left\{s_{i}(1), \ldots, s_{i}(m)\right\}} \lambda\left(\widehat{s}_{i}^{a_{i}}, s_{i}(m+1), s_{i}^{Z_{i} \backslash a_{i}}\right) \\
\eta \quad=\operatorname{Pr}\left(\widetilde{s}_{i}^{Z_{i} \backslash a_{i}}=s_{i}^{Z_{i} \backslash a_{i}} \mid s_{i}(m+1)\right)
\end{array}
$$

Since $s_{i}(m+1)$ is separated from $S_{i}^{a_{i}} \backslash\left\{s_{i}(1), \ldots, s_{i}(m)\right\}$, by exactly the same argument, we have $\lambda\left(s_{i}(m+1), \widehat{s}_{i}^{a_{i}}, s_{i}^{Z_{i} \backslash a_{i}}\right)=0$ for any $\widehat{s}_{i}^{a_{i}} \in S_{i}^{a_{i}} \backslash\left\{s_{i}(1), \ldots, s_{i}(m), s_{i}(m+1)\right\}$ and $s_{i}^{Z_{i} \backslash a_{i}}$. Thus, $\sum_{s_{i} Z_{i} \backslash a_{i}} \lambda\left(s_{i}(m+1), s_{i}(m+1), s_{i}^{Z_{i} \backslash a_{i}}\right)=1$ and $\lambda\left(\widehat{s}_{i}^{a_{i}}, s_{i}(m+1), s_{i}^{Z_{i} \backslash a_{i}}\right)=$ 0 for any $\widehat{s}_{i}^{a_{i}} \neq s_{i}(m+1)$ and $s_{i}^{Z_{i} \backslash a_{i}}$ by (13). This proves the proposition. 


\section{References}

1. Aoyagi, M.: Correlated types and Bayesian incentive compatible mechanisms with budget balance. J. Econ. Theory. 79, 142-151 (1998)

2. Arrow, K.: The property rights doctrine and demand revelation under incomplete information. In: Boskin M. (ed.) Economics and Human Welfare. New York, Academic Press (1979)

3. Bergemann, D., X. Shi, and J. Välimäki: Information acquisition in interdependent value auctions. J. Eur. Econ. Assoc. 7, 61-89 (2009)

4. Bergemann, D. and J. Välimäki: Information acquisition and mechanism design. Econometrica. 70, 1007-1033 (2002)

5. Bergemann, D. and J. Välimäki: The dynamic pivot mechanism. Econometrica. 78, 771789 (2010)

6. Bikhchandani, S.: Information acquisition and full surplus extraction. J. Econ. Theory. 145, 2282-2308 (2010)

7. Chen, Y-C. and S. Xiong: Genericity and robustness of full surplus extraction. Econometrica. 81, 825-847 (2013)

8. Clarke, E.: Multipart pricing of public goods. Public Choice. 9, 19-33 (1971)

9. Crémer, J. and R. McLean: Full extraction of surplus in Bayesian, dominant strategy auctions. Econometrica. 56, 1247-1258 (1988)

10. d'Aspremont, C. and L.A. Gérard-Varet: Incentives and incomplete information. J. Pub. Econ. 11, 25-45 (1979)

11. d'Aspremont, C. and L.A. Gérard-Varet: Bayesian incentive compatible beliefs. J. Math. Econ. 10, 83-103 (1982)

12. d'Aspremont, C., Crémer, J., and L.A. Gérard-Varet: Balanced Bayesian mechanisms. J. Econ. Theory. 115, 385-396 (2004)

13. Fang, H., and S. Morris: Multidimensional private value auctions. J. Econ. Theory. 126, $1-30(2006)$

14. Fudenberg, D., David Levine and Eric Maskin: The folk theorem with imperfect public information. Econometrica. 62, 997-1039 (1994)

15. Gershkov, A., and B. Szentes: Optimal voting schemes with costly information acquisition. J. Econ. Theory. 144, 36-68 (2009)

16. Gizatulina, A., and M. Hellwig: Beliefs, payoffs, information: On the robustness of the BDP property in models with endogenous beliefs. J. Math. Econ. 51, 136-153 (2014)

17. Groves, T.: Incentives in Teams. Econometrica. 41, 617-631 (1973)

18. Hausch, D. B., and L. Li: A common value auction model with endogenous entry and information acquisition. Econ. Theory. 3, No. 2, 315-334 (1993)

19. Heifetz, A. and Z. Neeman: On the generic (im)possibility of full surplus extraction in mechanism design. Econometrica. 74, 213-233 (2006)

20. Kandori, M.: Randomization, communication, and efficiency in repeated games with imperfect public monitoring. Econometrica. 71, 345-353 (2003)

21. Mas-Colell, A., M.D. Whinston, and J.R. Green: Microeconomic Theory. Oxford University Press (1995)

22. McLean, R. and A. Postlewaite: Informational size and incentive compatibility. Econometrica. 70, 2421-2453 (2002)

23. Neeman, Z.: The relevance of private information in mechanism design. J. Econ. Theory. 117,55-77 (2004)

24. Obara, I.: The full surplus extraction theorem with hidden actions. Adv. Theor. Econ. 8, No. 1, Article 8, http://www.bepress.com/bejte/vol8/iss1/art8/ (2008)

25. Parreiras, S.O.: Correlated information, mechanism design and informational rents. J. Econ. Theory. 123, 210-217 (2005)

26. Rahman, D. and I. Obara: Mediated partnerships. Econometrica. 78, 285-308 (2010)

27. Stegeman, M.: Participation costs and efficient auctions. J. Econ. Theory. 71, 228-259 (1996)

28. Vickrey, W.: Counterspeculation, auctions, and competitive sealed tenders. J. Finance. 16, 8-37 (1961) 